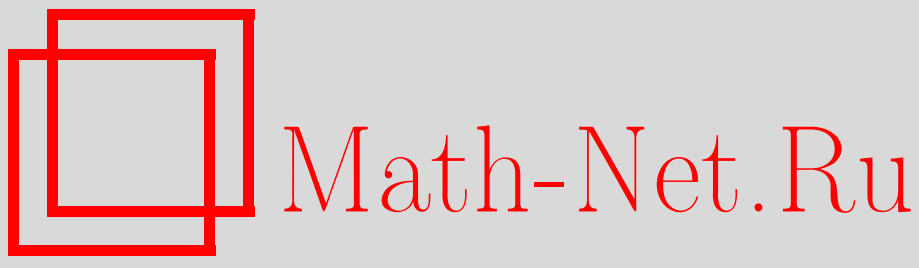

А. М. Рекало, И. Д. Чуешов, Глобальный аттрактор контактной параболической задачи в тонкой двухслойной области, Матем. сб., 2004, том 195, номер 1, 103-128

DOI: https://doi.org/10.4213/sm795

Использование Общероссийского математического портала Math-Net.Ru подразумевает, что вы прочитали и согласны с пользовательским соглашением http://www . mathnet.ru/rus/agreement

Параметры загрузки:

IP: 3.80 .253 .173

26 апреля 2023 г., 14:59:31 
УДК 517.94

\author{
А.М. Рекало, И. Д. Чуешов
}

\title{
Глобальный аттрактор контактной параболической задачи в тонкой двухслойной области
}

Рассматривается полулинейное параболическое уравнение в объединении двух примыкающих друг к другу по основаниям тонких ограниченных цилиндрических областей $\Omega_{1, \varepsilon}=\Gamma \times(0, \varepsilon)$ и $\Omega_{2, \varepsilon}=\Gamma \times(-\varepsilon, 0)$, где $\Gamma-$ область в $\mathbb{R}^{d}$, $d \leqslant 3$. Неизвестные функции связываются некоторым условием сопряжения на общем основании $\Gamma$. Данная задача может служить моделью химической кинетики, описьвающей поведение системы, состоящей из двух компонентов, реагирующих на границе. Предполагается, что интенсивность реакции зависит от $\varepsilon$ - поперечного размера области - и имеет порядок $\varepsilon^{\alpha}$.

В работе исследуются предельные свойства эволюционной полугрупшы $S_{\alpha, \varepsilon}(t)$, порожденной исходной задачей, при $\varepsilon \rightarrow 0$ (т.е. при утончении исходной области). Показано, что эти свойства существенно зависят от величины показателя $\alpha$. В зависимости от того, будет ли $\alpha$ принимать значение 1 , больше 1 или меньше 1 , асимптотическим пределом исходной задачи будут служить три различные системы уравнений на Г. При естественных предположениях устанавливаются свойства непрерьвности глобального аттрактора полугрупшы $S_{\alpha, \varepsilon}(t)$ при $\varepsilon \rightarrow 0$.

Библиографиия: 17 названий.

\section{Введение}

Пусть $\Omega_{1, \varepsilon}$ и $\Omega_{2, \varepsilon}-$ ограниченные области в $\mathbb{R}^{d+1}, d \leqslant 3$, вида

$$
\Omega_{1, \varepsilon}=\Gamma \times(0, \varepsilon), \quad \Omega_{2, \varepsilon}=\Gamma \times(-\varepsilon, 0),
$$

где $0<\varepsilon \leqslant 1$ и Г- ограниченная область в $\mathbb{R}^{d}$ с границей $\partial Г$ класса $C^{2}$. В дальнейшем для простоты обозначений множества $\Gamma \times\{0\} \subset \mathbb{R}^{d+1}$ и $\Gamma \subset \mathbb{R}^{d}$ не различаются. Кроме того, ниже для точки $x \in \Omega_{i, \varepsilon}$ используется обозначение $x=\left(x^{\prime}, x_{d+1}\right)$, где $x^{\prime} \in \Gamma$ и $x_{d+1} \in(0, \varepsilon)$ или $x_{d+1} \in(-\varepsilon, 0)$.

Рассматривается система полулинейных параболических уравнений

$$
\partial_{t} w_{i}=\nu_{i} \Delta w_{i}-a w_{i}+f_{i}\left(w_{i}\right)+h_{i}(x), \quad t>0, \quad x \in \Omega_{i, \varepsilon}, \quad i=1,2,
$$

с начальными условиями

$$
w_{i}(0, x)=w_{i, 0}(x), \quad x \in \Omega_{i, \varepsilon}, \quad i=1,2 .
$$

Работа вьполнена при частичной поддержке фонда INTAS (грант № 2000/899). 
Предполагается, что компоненты $w_{1}$ и $w_{2}$ удовлетворяют условию Неймана

$$
\left.\frac{\partial w_{i}}{\partial n_{i}}\right|_{\partial \Omega_{i, \varepsilon} \backslash \Gamma}=0, \quad i=1,2
$$

на внешней части границы составной области $\Omega_{\varepsilon}=\Omega_{1, \varepsilon} \cup \Omega_{2, \varepsilon}$ И условию сопряжения на Г вида

$$
\begin{aligned}
& \left.\left(\nu_{1} \frac{\partial w_{1}}{\partial n_{1}}+\varepsilon^{\alpha} k\left(x^{\prime}\right)\left(w_{1}-w_{2}\right)\right)\right|_{\Gamma}=0, \\
& \left.\left(\nu_{2} \frac{\partial w_{2}}{\partial n_{2}}+\varepsilon^{\alpha} k\left(x^{\prime}\right)\left(w_{2}-w_{1}\right)\right)\right|_{\Gamma}=0 .
\end{aligned}
$$

Здесь $\partial w_{i} / \partial n_{i}$ обозначает производную функции по направлению нормали, внешней к $\partial \Omega_{i, \varepsilon}$. Параметры $\nu_{i}$ и $а$ считаются положительными. Относительно функций $f_{i}$ предполагается, что

$$
f_{i}(v) \in C^{2}(\mathbb{R}), \quad\left|f^{\prime \prime}(v)\right| \leqslant M\left(1+|v|^{p}\right),
$$

где $0 \leqslant p \leqslant(3-d) /(d-1)$ при $d=2,3$ и $p \geqslant 0$ любое при $d=1$. Кроме того, для любого $\delta>0$ сушествует $C_{\delta}>0$ такое, что

$$
v f_{i}(v) \leqslant \delta v^{2}+C_{\delta}
$$

и найдутся такие $\beta>0, \eta>0$ и $\gamma<a$, что

$$
v f_{i}(v)-\beta \int_{0}^{v} f_{i}(w) d w \leqslant \gamma v^{2}+\eta
$$

Предполагается также, что

$$
h_{i}(x) \in H^{1}\left(\Omega_{i, 1}\right), \quad i=1,2,
$$

и

$$
k\left(x^{\prime}\right) \in C^{1}(\Gamma), \quad \varkappa=\inf _{x^{\prime} \in \Gamma} k\left(x^{\prime}\right)>0 .
$$

Задача (1)-(4) представляет собой модель химической кинетики, описывающей поведение системы, состоящей из двух компонентов, заполняюших тонкие соприкасающиеся слои $\Omega_{1, \varepsilon}$ и $\Omega_{2, \varepsilon}$, разделенные проницаемой мембраной Г. Взаимодействие (реакция) компонентов происходит только на поверхности Г. Интенсивность реакции на $Г$ зависит от толщины областей, заполненных реагентами, и описывается коэффициентом $\varepsilon^{\alpha} k\left(x^{\prime}\right)$.

В данной работе изучаются предельные свойства решений задачи (1)-(4) при $\varepsilon \rightarrow 0$ (т.е. при утончении области $\Omega_{\varepsilon}$ ). Эти свойства сушественно зависят от величины показателя $\alpha$, входящего в коэффициент обмена $\varepsilon^{\alpha} k\left(x^{\prime}\right)$. При указанных выше условиях задача (1)-(4) порождает эволюционную полугруппу $T_{\alpha, \varepsilon}(t)$, $t \geqslant 0$, в пространстве $H^{1}\left(\Omega_{1, \varepsilon}\right) \oplus H^{1}\left(\Omega_{2, \varepsilon}\right)$, обладающую компактным глобальным 
аттрактором $\mathscr{M}_{\alpha, \varepsilon}$ (см. теорему 1.1). Работа посвящена изучению свойств аттрактора $\mathscr{M}_{\alpha, \varepsilon}$ при $\varepsilon \rightarrow 0$. Оказывается, что в зависимости от того, будет ли параметр $\alpha$ принимать значение 1 , больше 1 или меньше $<1$, "асимптотическим пределом" $\mathscr{M}_{\alpha, \varepsilon}$ при $\varepsilon \rightarrow 0$ будет аттрактор эволюционной полугруппы, порождаемой следующими уравнениями в области $Г$ соответственно:

а) при $\alpha=1$ :

$$
\left\{\begin{array}{l}
\partial_{t} v_{i}=\nu_{i} \Delta v_{i}+(-1)^{i} k(x)\left(v_{1}-v_{2}\right)-a v_{i}+f_{i}\left(v_{i}\right)+h_{i}\left(x^{\prime}, 0\right), \quad t>0, x^{\prime} \in \Gamma \\
\left.\frac{\partial v_{i}}{\partial n}\right|_{\partial \Gamma}=0,\left.v_{i}\right|_{t=0}=v_{i, 0}, \quad i=1,2
\end{array}\right.
$$

б) при $\alpha>1$ :

$$
\left\{\begin{array}{l}
\partial_{t} v_{i}=\nu_{i} \Delta v_{i}-a v_{i}+f_{i}\left(v_{i}\right)+h_{i}\left(x^{\prime}, 0\right), \quad t>0, x^{\prime} \in \Gamma \\
\left.\frac{\partial v_{i}}{\partial n}\right|_{\partial \Gamma}=0,\left.v_{i}\right|_{t=0}=v_{i, 0}, \quad i=1,2
\end{array}\right.
$$

в) при $\alpha<1$ :

$$
\left\{\begin{array}{l}
\partial_{t} v=\nu \Delta v-a v+f(v)+h\left(x^{\prime}\right), \quad t>0, x^{\prime} \in \Gamma \\
\left.\frac{\partial v}{\partial n}\right|_{\partial \Gamma}=0,\left.v\right|_{t=0}=v_{0}
\end{array}\right.
$$

где $\nu=\frac{1}{2}\left(\nu_{1}+\nu_{2}\right)$,

$$
f(v)=\frac{1}{2}\left(f_{1}(v)+f_{2}(v)\right), \quad h\left(x^{\prime}\right)=\frac{1}{2}\left(h_{1}\left(x^{\prime}, 0\right)+h_{2}\left(x^{\prime}, 0\right)\right) .
$$

Отметим, что задачи об асимптотическом поведении решений нелинейных дифференциальных уравнений в тонких (однослойных) областях рассматривались многими авторами (см., например, [1]-[4] и представленную там библиографию). Кроме того, имеется значительное число работ (см., например, [5]-[12] и ссылки, приведенные в этих работах), посвлшенных обсуждению вопроса о непрерывности аттракторов относительно сингулярных возмушений для различных классов диссипативных динамических систем.

Опишем структуру работы. В следуюшем $§ 1$ приведены точные формулировки основных результатов. Параграф 2 носит предварительньй характер. В нем, в частности, вводится вспомогательная (масштабированная) задача, изучаются свойства ее эллиптической части и устанавливаются равномерные оценки для ее глобального аттрактора. В $\S 3$ доказьвается сходимость эволюционных операторов. Заключительньй $\S 4$ посвящен доказательству основных результатов об асимптотическом поведении глобального аттрактора задачи (1)-(4). 


\section{§1. Основные результаты}

Пусть

$$
\mathscr{F}_{\varepsilon}=H^{1}\left(\Omega_{1, \varepsilon}\right) \oplus H^{1}\left(\Omega_{2, \varepsilon}\right), \quad \mathscr{F}_{0}=H^{1}(\Gamma) \oplus H^{1}(\Gamma), \quad \mathbf{L}^{2}=L^{2}(\Gamma) \oplus L^{2}(\Gamma) .
$$

Здесь и в дальнейшем $H^{s}(\mathscr{O})$ - соболевскоепространство на множестве $\mathscr{O}$. Нормы в пространствах $\mathscr{F}_{\varepsilon}, \mathscr{F}_{0}$ и $\mathbf{L}^{2}$ обозначаются $\|\cdot\|_{\mathscr{F}_{\varepsilon}},\|\cdot\|_{\mathscr{F}_{0}}$ и $\|\cdot\|_{\mathbf{L}^{2}}$ соответственно.

Для формулировки основных результатов нам потребуется следующее предварительное утверждение.

Teорема 1.1. В пространстве $\mathscr{F}_{\varepsilon}$ задача (1)-(4) порождает компактную диссипативную систему $\left(\mathscr{F}_{\varepsilon}, T_{\alpha, \varepsilon}(t)\right)$, обладающую компактныц глобальныцм аттрактором $\mathscr{M}_{\alpha, \varepsilon}$, лежащим в $H^{2}\left(\Omega_{1, \varepsilon}\right) \oplus H^{2}\left(\Omega_{2, \varepsilon}\right)$. Эволюционный оператор $T_{\alpha, \varepsilon}(t)$ определяется формулой

$$
T_{\alpha, \varepsilon}(t)\left(w_{1,0} ; w_{2,0}\right)=\left(w_{1}(t) ; w_{2}(t)\right)
$$

где пара $\left(w_{1}(t) ; w_{2}(t)\right)$ является решением задачи (1)-(4).

Кроме того, задачи (10) (случай $\alpha=1)$ и (11) (случай $\alpha>1)$ в пространстве $\mathscr{F} 0$ порождают компактные диссипативные системы с глобальными аттракторами $\mathscr{A}_{\alpha}$, лежащими в $H^{2}(\Gamma) \oplus H^{2}(\Gamma)$.

Задача $(12)$ (случай $\alpha<1)$ порождает компактную диссипативную систему в $H^{1}(\Gamma)$, обладающую глобальным аттрактором $\widetilde{\mathscr{A}} \subset H^{2}(\Gamma)$.

Напомним (см., например, [5], [6], [13]), что под компактной динамической системой понимается пара $(H, S(t))$, состояшая из метрического пространства $H$ и (непрерывного) эволюционного оператора $S(t)$ и обладающая компактным поглощающим множеством (множество $B_{0} \subset H$ называется поглощающим для $(H, S(t))$, если для любого ограниченного множества $B \subset H$ сушествует момент времени $t_{B} \geqslant 0$ такой, что $S(t) B \subset B_{0}$ для всех $\left.t \geqslant t_{B}\right)$. Глобальнымм аттрактором динамической системы $(H, S(t))$ назьвается ограниченное замкнутое множество $\mathscr{A} \subset H$ такое, что $S(t) \mathscr{A}=\mathscr{A}$ при $t \geqslant 0$ и

$$
\lim _{t \rightarrow+\infty} \sup _{v \in B} \operatorname{dist}_{H}(S(t) v, \mathscr{A})=0
$$

для любого ограниченного множества $B \subset H$.

Доказательство теоремы 1.1 носит стандартньй характер и получается дословным повторением (с незначительными изменениями) соответствующих рассуждений, приведенных в [5] (см. также [6], [13]).

Для описания предельного поведения эволюционных операторов $T_{\alpha, \varepsilon}$ и глобальных аттракторов $\mathscr{M}_{\alpha, \varepsilon}$ введем операторы усреднения вдоль направления $x_{d+1}$ :

$$
M_{1}^{\varepsilon} u_{1}=\frac{1}{\varepsilon} \int_{0}^{\varepsilon} u_{1}\left(x^{\prime}, y\right) d y, \quad M_{2}^{\varepsilon} u_{2}=\frac{1}{\varepsilon} \int_{-\varepsilon}^{0} u_{2}\left(x^{\prime}, y\right) d y .
$$

Пусть $M^{\varepsilon} u=\left(M_{1}^{\varepsilon} u_{1} ; M_{2}^{\varepsilon} u_{2}\right)$. Ясно, что $M^{\varepsilon}$-непрерьвный оператор из $L^{2}\left(\Omega_{1, \varepsilon}\right) \oplus$ $L^{2}\left(\Omega_{2, \varepsilon}\right)$ в $\mathbf{L}^{2}$ и из $\mathscr{F}_{\varepsilon}$ в $\mathscr{F}_{0}$.

Следующее утверждение описывает асимптотическое поведение оператора $T_{\alpha, \varepsilon}(t)$ при $\varepsilon \rightarrow 0$ в случае $\alpha \geqslant 1$. 
TЕОРема 1.2. Пусть $\alpha \geqslant 1 u h(\varepsilon)=\|h\|_{\mathscr{F}_{\varepsilon}}^{2} \equiv\left\|h_{1}\right\|_{H^{1}\left(\Omega_{1, \varepsilon}\right)}^{2}+\left\|h_{2}\right\|_{H^{1}\left(\Omega_{2, \varepsilon}\right)}^{2}$. Обозначим через $S_{\alpha}(t)$ эволючионный оператор задачи (10) при $\alpha=1$ u (11) при $\alpha>1$ соответственно. Тогда для каждого $r>0$ существуют констанmъь $c_{1}(r)$ и $c_{2}(r)$ такие, что

$$
\begin{gathered}
\left\|M^{\varepsilon} T_{\alpha, \varepsilon}(t) w_{0}-S_{\alpha}(t) M^{\varepsilon} w_{0}\right\|_{\mathbf{L}^{2}}^{2}+\int_{0}^{t}\left\|M^{\varepsilon} T_{\alpha, \varepsilon}(\tau) w_{0}-S_{\alpha}(\tau) M^{\varepsilon} w_{0}\right\|_{\mathscr{F}_{0}}^{2} d \tau \\
\leqslant c_{1}(r)\left(\varepsilon^{4}+\varepsilon h(\varepsilon)\right) e^{c_{2}(r) t}
\end{gathered}
$$

для любого элемента $w_{0}=\left(w_{1,0} ; w_{2,0}\right) \in \mathscr{F}_{\varepsilon}$ такого, что $\varepsilon^{-1}\left\|w_{0}\right\|_{\mathscr{F} \varepsilon}^{2} \leqslant r$. Кроме того, справедлива оченка

$$
\left\|M^{\varepsilon} T_{\alpha, \varepsilon}(t) w_{0}-S_{\alpha}(t) M^{\varepsilon} w_{0}\right\|_{H^{s}(\Gamma) \oplus H^{s}(\Gamma)} \leqslant c_{1}(r)\left(\varepsilon^{4}+\varepsilon h(\varepsilon)\right)^{(1-s) / 2} e^{c_{2}(r) t}
$$

для любого $0 \leqslant s<1$.

Отметим, что с подходящей константой $r$ условие $\varepsilon^{-1}\left\|w_{0}\right\|_{\mathscr{F}}^{2} \leqslant r$ выполнено для любой функции $w_{0}$ из $C^{1}\left(\bar{\Omega}_{1, \varepsilon}\right) \oplus C^{1}\left(\bar{\Omega}_{2, \varepsilon}\right)$.

Утверждение о сходимости решений, которое удается установить в случае $\alpha<1$, является более слабым по сравнению с теоремой 1.2 .

ТЕОРема 1.3. Пусть $\alpha<1 u\left(w_{1}^{\varepsilon}(t) ; w_{2}^{\varepsilon}(t)\right)$ - решение задачи (1)-(4) с начальными условиями $w_{1,0}\left(x^{\prime}, x_{d+1}\right)=v_{0}\left(x^{\prime}\right)$ u $w_{2,0}\left(x^{\prime}, x_{d+1}\right)=v_{0}\left(x^{\prime}\right)$, где $v_{0}-$ произвольный элемент из $H^{1}(\Gamma)$. Тогда

$$
\lim _{\varepsilon \rightarrow 0} \sum_{i=1,2} \sup _{t \in[0, T]}\left\|M_{i}^{\varepsilon} w_{i}^{\varepsilon}(t)-v(t)\right\|_{H^{s}(\Gamma)}=0
$$

для любого $0 \leqslant s<1$, где $v(t)$ - решение задачи (12).

Основными результатами работы являются следующие теоремы о близости аттракторов допредельной и предельных задач.

ТЕОремА 1.4. Для любых $\alpha \geqslant 1$ справедливо соотношение

$$
\lim _{\varepsilon \rightarrow 0} \sup _{u \in \mathscr{M}_{\alpha, \varepsilon}} \inf _{v \in \mathscr{A}_{\alpha}}\left\|M^{\varepsilon} u-v\right\|_{H^{1}(\Gamma) \oplus H^{1}(\Gamma)}=0
$$

где $\mathscr{M}_{\alpha, \varepsilon}$ - глобальный аттрактор задачи (1)-(4) и $\mathscr{A}_{\alpha}-$ глобальный аттрактор задачи (10) при $\alpha=1$ и (11) при $\alpha>1$ соответственно.

ТеОрема 1.5. Пусть $\alpha<1$. Тогда выполняется соотношение (16) для $\mathscr{A}_{\alpha}$, имеющего вид

$$
\mathscr{A}_{\alpha}=\left\{(v ; v) \in H^{1}(\Gamma) \oplus H^{1}(\Gamma): v \in \tilde{\mathscr{A}}\right\}
$$

әде $\tilde{\mathscr{A}}$ - глобальный аттрактор задачи (12).

Теоремы 1.4 и 1.5 утверждают полунепрерывность аттракторов $\mathscr{M}_{\alpha, \varepsilon}$ сверху при $\varepsilon \rightarrow 0$. В случае, когда аттрактор соответствующей предельной задачи регулярен (ситуация “общего положения", см., например, [5] и [13]), можно также установить полную непрерывность (в симметризованной метрике Хаусдорфа) семейства $\left\{\mathscr{M}_{\alpha, \varepsilon}\right\}_{\varepsilon \in(0,1]}$ при $\varepsilon \rightarrow 0$. А именно справедливо следующее утверждение. 
Теорема 1.6. Пусть $\alpha \geqslant 1$. Пусть все неподвижные точки задачи (10) при $\alpha=1$ ((11) при $\alpha>1)$ гиперболические. Тогда для некоторых $C, \varepsilon_{0}$, $\sigma>0$ справедливо соотночение

$$
\operatorname{dist}\left(M^{\varepsilon} \mathscr{M}_{\alpha, \varepsilon} \mid \mathscr{A}_{\alpha}\right)+\operatorname{dist}\left(\mathscr{A}_{\alpha} \mid \mathscr{M}_{\alpha, \varepsilon}\right) \leqslant C \varepsilon^{\sigma}, \quad 0<\varepsilon \leqslant \varepsilon_{0},
$$

где $\mathscr{M}_{\alpha, \varepsilon}$ и $\mathscr{A}_{\alpha}-$ те же что и в теореме 1.4 , а расстояние $\operatorname{dist}(\mathscr{A} \mid \mathscr{M})$ от множсества $\mathscr{A}$ до множества $\mathscr{M}$ определено равенством

$$
\operatorname{dist}(\mathscr{A} \mid \mathscr{M})=\sup _{v \in \mathscr{A}} \inf _{u \in \mathscr{M}}\left\|M^{\varepsilon} u-v\right\|_{H^{1}(\Gamma) \oplus H^{1}(\Gamma)}
$$

Таким образом, теоремы 1.4 и 1.6 показывают, что случай $\alpha>1$ отвечает слабому (исчезаюшему в пределе $\varepsilon \rightarrow 0$ ) взаимодействию компонентов $u_{1}^{\varepsilon}(t)$ и $u_{2}^{\varepsilon}(t)$ решения задачи (1)-(4). Их предельная динамика описывается парой независимых уравнений (11) и асимптотически эти компоненты эволюционируют независимо друг от друга. При $\alpha=1$ предельная задача (10) содержит обменный член $k\left(x^{\prime}\right)\left(v_{1}-v_{2}\right)$, т.е. в пределе $\varepsilon \rightarrow 0$ компоненты $u_{1}^{\varepsilon}(t)$ и $u_{2}^{\varepsilon}(t)$ продолжают взаимодействовать.

Теорема 1.5 означает, что случай $\alpha<1$ отвечает сильному предельному взаимодействию компонентов. В пределе $\varepsilon \rightarrow 0$ наблюдается синхронизация компонентов $u_{1}^{\varepsilon}(t)$ и $u_{2}^{\varepsilon}(t)$. Фактически, в пределе возникает смесь компонентов (со средними коэффициентом диффузии $\nu$ и реактивным членом $f(u)$ ). Отметим также, что асимптотическое поведение решений задачи (1)-(4) при условиях $\alpha=0, \nu_{1}=\nu_{2}$, $f_{1}(u)=f_{2}(u), h_{1}=h_{2}=0, d=1$ изучалось в работе [14]. В этой работе, в частности, было доказано, что при достаточно малых $\varepsilon$ аттрактор $\mathscr{M}_{0, \varepsilon}$ совпадает с множеством $\mathscr{A}_{\alpha}$, определяемьм соотношением (17).

Отметим также, что теоремы 1.4 и 1.6 остаются в силе, если вместо оператора усреднения $M^{\varepsilon}$ взять оператор следа $\gamma$, действующий по формуле $\gamma\left(u_{1} ; u_{2}\right)=$ $\left(u_{1}\left(x^{\prime},+0\right) ; u_{2}\left(x^{\prime},-0\right)\right)$ (см. замечание 4.2$)$. То же самое относится и к теореме 1.5 , однако вместо соболевской $H^{1}$-нормы нужно рассматривать $L^{2}$-норму.

\section{§2. Предварительные рассмотрения}

2.1. Масштабирование и функциональные пространства. Для доказательства приведенных выше теорем удобно иметь дело с фиксированной областью, в которой определены все уравнения при $\varepsilon>0$. Введем новые координаты $(x, y) \in \mathbb{R}^{d+1}$ следующим образом:

$$
x=x^{\prime}, \quad x \in \Gamma, \quad y=\varepsilon^{-1} x_{d+1}, \quad y \in(-1,1) .
$$

При этом область $\Omega_{\varepsilon}$ перейдет в $\Omega=\Omega_{1} \cup \Omega_{2}$, где $\Omega_{1}=\Gamma \times(0,1), \Omega_{2}=\Gamma \times(-1,0)$, оператор $\nabla=\left(\nabla_{x^{\prime}}, \partial_{x_{d+1}}\right)-$ в $\nabla_{\varepsilon}=\left(\nabla_{x}, \varepsilon^{-1} \partial_{y}\right), \Delta=\Delta_{x^{\prime}}+\partial_{x_{d+1}}^{2}-$ в $\Delta_{\varepsilon}=\Delta_{x}+$ $\varepsilon^{-2} \partial_{y y}$. Задаче (1)-(4) будет соответствовать задача

$$
\partial_{t} u_{i}=\nu_{i} \Delta_{\varepsilon} u_{i}-a u_{i}+f_{i}\left(u_{i}\right)+h_{i}^{\varepsilon}(x, y), \quad t>0, \quad(x, y) \in \Omega_{i}, \quad i=1,2,
$$


с начальными данными

$$
u_{i}(0, x, y)=u_{i, 0}(x, y), \quad(x, y) \in \Omega_{i}, \quad i=1,2
$$

и граничными условиями

$$
\begin{gathered}
\left.\frac{\partial u_{i}}{\partial n_{i}}\right|_{\partial \Omega_{i} \backslash \Gamma}=0, \quad i=1,2 \\
\left.\left(\nu_{i} \frac{\partial u_{i}}{\partial y}-\varepsilon^{\alpha+1} k(x)\left(u_{1}-u_{2}\right)\right)\right|_{y=0}=0, \quad i=1,2 .
\end{gathered}
$$

Здесь $h_{i}^{\varepsilon}(x, y)=h_{i}(x, \varepsilon y)$ и $n_{i}$ - внешняя нормаль к границе $\partial \Omega_{i}$. Решение $w\left(t, x^{\prime}, x_{d+1}\right)$ задачи (1)-(4) выражается через решение $u(t, x, y)$ задачи $(20)-(23)$ по формуле $w\left(t, x^{\prime}, x_{d+1}\right)=u\left(t, x^{\prime}, \varepsilon^{-1} x_{d+1}\right)$.

Введем пространство

$$
H=L^{2}\left(\Omega_{1}\right) \oplus L^{2}\left(\Omega_{2}\right) \simeq L^{2}(\Omega)
$$

с нормой $\|u\|^{2} \equiv\left\|u_{1}\right\|_{L^{2}\left(\Omega_{1}\right)}^{2}+\left\|u_{2}\right\|_{L^{2}\left(\Omega_{2}\right)}^{2}$, где $u=\left(u_{1} ; u_{2}\right),\left.u_{i} \equiv u\right|_{\Omega_{i}}$, и определим семейство классов Соболева

$$
H_{\varepsilon}^{1}=H^{1}\left(\Omega_{1}\right) \oplus H^{1}\left(\Omega_{2}\right), \quad \varepsilon \in(0,1],
$$

с нормами

$$
\|u\|_{1, \varepsilon}^{2} \equiv \sum_{i=1}^{2}\left(\left\|u_{i}\right\|_{H^{1}\left(\Omega_{i}\right)}^{2}+\varepsilon^{-2}\left\|\partial_{y} u_{i}\right\|_{L^{2}\left(\Omega_{i}\right)}^{2}\right) .
$$

Всякий элемент $v \in H^{1}(\Gamma) \oplus H^{1}(\Gamma)$ естественным образом продолжается до элемента $u \in H_{\varepsilon}^{1}$ по формуле $u_{i}(x, y) \equiv v_{i}(x),(x, y) \in \Omega_{i}, i=1,2$; возможность этого продолжения будет предполагаться в дальнейшем без дополнительных оговорок.

2.2. Форма Дирихле. На пространстве $H_{\varepsilon}^{1}=H^{1}\left(\Omega_{1}\right) \oplus H^{1}\left(\Omega_{2}\right)$ рассмотрим билинейную форму

$$
\begin{aligned}
a_{\alpha, \varepsilon}(u, v)= & \sum_{i=1}^{2} \nu_{i}\left(\left(\nabla_{x} u_{i}, \nabla_{x} v_{i}\right)_{L^{2}\left(\Omega_{i}\right)}+\frac{1}{\varepsilon^{2}}\left(\partial_{y} u_{i}, \partial_{y} v_{i}\right)_{L^{2}\left(\Omega_{i}\right)}\right)+a \cdot(u, v)_{H} \\
& +\varepsilon^{\alpha-1} \int_{\Gamma} k(x)\left(u_{1}(x, 0)-u_{2}(x, 0)\right)\left(v_{1}(x, 0)-v_{2}(x, 0)\right) d x,
\end{aligned}
$$

где $u=\left(u_{1} ; u_{2}\right)$ и $v=\left(v_{1} ; v_{2}\right)$ - элементы из $H_{\varepsilon}^{1}$. Форма $a_{\alpha, \varepsilon}(u, v)$ симметрична и удовлетворяет в силу (9) оценкам

$$
c_{1}\|u\|_{1, \varepsilon}^{2} \leqslant a_{\alpha, \varepsilon}(u, u) \leqslant c_{2}\left(1+\varepsilon^{\alpha-1}\right)\|u\|_{1, \varepsilon}^{2}, \quad u \in H_{\varepsilon}^{1} .
$$


Здесь и далее мы опускаем индекс $\varepsilon$ у постоянных, которые можно выбрать независимо от $\varepsilon \in(0,1]$.

Можно показать, что форма $a_{\alpha, \varepsilon}(u, v)$ замкнутав $H$. Поэтому существует такой однозначно определенный положительный самосопряженный оператор $A_{\alpha, \varepsilon}$, что $\mathrm{D}\left(A_{\alpha, \varepsilon}\right) \subset H_{\varepsilon}^{1}$ и

$$
a_{\alpha, \varepsilon}(u, v)=\left(A_{\alpha, \varepsilon} u, v\right)_{H}, \quad u \in \mathrm{D}\left(A_{\alpha, \varepsilon}\right), \quad v \in H_{\varepsilon}^{1} .
$$

Можно показать, что область определения $\mathrm{D}\left(A_{\alpha, \varepsilon}\right)$ состоит из элементов $u \in H_{\varepsilon}^{2}$, удовлетворяющих граничным условиям (22) и (23), причем

$$
A_{\alpha, \varepsilon} u=\left(-\nu_{1} \Delta_{\varepsilon} u_{1}+a u_{1} ;-\nu_{2} \Delta_{\varepsilon} u_{2}+a u_{2}\right), \quad u=\left(u_{1}, u_{2}\right) \in \mathrm{D}\left(A_{\alpha, \varepsilon}\right) .
$$

Кроме того, $\mathrm{D}\left(A_{\alpha, \varepsilon}^{1 / 2}\right)=H_{\varepsilon}^{1}$ и $a_{\alpha, \varepsilon}(u, u)=\left\|A_{\alpha, \varepsilon}^{1 / 2} u\right\|^{2}$.

Определим в $H$ ортогональньй проектор $M u=\left(M_{1} u_{1}, M_{2} u_{2}\right)$, где

$$
M_{1} u_{1}=\int_{0}^{1} u_{1}(x, y) d y, \quad M_{2} u_{2}=\int_{-1}^{0} u_{2}(x, y) d y .
$$

Положим $N u=\left(N_{1} u_{1} ; N_{2} u_{2}\right)=(I-M) u$. В дальнейшем постоянно будем использовать неравенства

$$
\begin{gathered}
\|u-M u\|_{L^{2}(\Omega)} \leqslant\left(\sum_{i=1}^{2}\left\|\partial_{y} N_{i} u_{i}\right\|_{L^{2}\left(\Omega_{i}\right)}^{2}\right)^{1 / 2} \leqslant \varepsilon\|N u\|_{1, \varepsilon}, \quad u \in H_{\varepsilon}^{1}, \\
\left\|u_{i}(x, 0)-M_{i} u_{i}\right\|_{L^{2}(\Gamma)} \leqslant\left\|\partial_{y} N_{i} u_{i}\right\|_{L^{2}\left(\Omega_{i}\right)} \leqslant \varepsilon\|N u\|_{1, \varepsilon}, \quad u \in H_{\varepsilon}^{1} .
\end{gathered}
$$

Следуюшее утверждение описывает поведение оператора $A_{\alpha, \varepsilon}^{-1}$ при $\varepsilon \rightarrow 0$ в случае, когда $\alpha \geqslant 1$.

Лемма 2.1. Пусть $\alpha \geqslant 1$. Тогда существует постоянная $C$ такая, что

$$
\left\|\left(A_{\alpha, \varepsilon}^{-1}-A_{\alpha}^{-1}\right) v\right\|_{1, \varepsilon} \leqslant C\left(\delta_{\alpha} \varepsilon+\left(1-\delta_{\alpha}\right) \varepsilon^{\alpha-1}\right)\|v\|, \quad v \in M H
$$

где $\delta_{\alpha}=1$ при $\alpha=1$ и $\delta_{\alpha}=0$ при $\alpha>1$, а $A_{\alpha}$ - положительный самосопряженный оператор в $L^{2}(\Gamma) \oplus L^{2}(\Gamma)$ вида

$$
\left(A_{\alpha} v\right)_{i}=-\nu_{i} \Delta_{x} v_{i}-(-1)^{i} \delta_{\alpha} k(x)\left(v_{1}-v_{2}\right)+a v_{i}, \quad i=1,2
$$

с областью определения

$$
D\left(A_{\alpha}\right)=\left\{v \in H^{2}(\Gamma) \oplus H^{2}(\Gamma):\left.\frac{\partial v}{\partial n}\right|_{\partial \Gamma}=0\right\} .
$$


ДокАЗАТЕЛЬСтво. Рассмотрим только случай $\alpha=1$ (при $\alpha>1$ рассуждения аналогичны). В дальнейшем для краткости полагаем $a_{\varepsilon} \equiv a_{\alpha, \varepsilon}, A_{\varepsilon} \equiv A_{1, \varepsilon}$ и $A \equiv A_{1}$. Оператору $A$ отвечает форма

$$
\begin{aligned}
a(u, v)= & \sum_{i=1}^{2}\left(\nu_{i}\left(\nabla_{x} u_{i}, \nabla_{x} v_{i}\right)_{L^{2}(\Gamma)}+a \cdot\left(u_{i}, v_{i}\right)_{L^{2}(\Gamma)}\right) \\
& +\int_{\Gamma} k(x)\left(u_{1}(x)-u_{2}(x)\right)\left(v_{1}(x)-v_{2}(x)\right) d x
\end{aligned}
$$

где $u=\left(u_{1} ; u_{2}\right)$ и $v=\left(v_{1} ; v_{2}\right)$ - элементы из $H^{1}(\Gamma) \oplus H^{1}(\Gamma)$. При этом легко видеть, что

$$
a_{\varepsilon}(u, w)=a(M u, w)-\sum_{i=1}^{2}(-1)^{i} \int_{\Gamma} k(x)\left(u_{i}(x, 0)-M_{i} u_{i}\right)\left(w_{1}-w_{2}\right) d x
$$

для всех $u \in H_{\varepsilon}^{1}$ и $w \in H^{1}(\Gamma) \oplus H^{1}(\Gamma)$. Поэтому, используя очевидное равенство

$$
a_{\varepsilon}\left(u, A_{\varepsilon}^{-1} v\right)=(u, v)_{H}=(M u, v)_{L^{2}(\Gamma) \oplus L^{2}(\Gamma)}=a\left(M u, A^{-1} v\right)
$$

для всех $u \in H_{\varepsilon}^{1}, v \in M H$ получаем, что

$a_{\varepsilon}\left(u,\left(A_{\varepsilon}^{-1}-A^{-1}\right) v\right)=\sum_{i=1}^{2}(-1)^{i} \int_{\Gamma} k(x)\left(u_{i}(x, 0)-M_{i} u_{i}\right)\left(\left(A^{-1} v\right)_{1}-\left(A^{-1} v\right)_{2}\right) d x$.

Следовательно, используя (26), получаем, что

$$
\left|a_{\varepsilon}\left(u,\left(A_{\varepsilon}^{-1}-A^{-1}\right) v\right)\right| \leqslant C \varepsilon\|u\|_{1, \varepsilon}\left\|A^{-1} v\right\| .
$$

Взяв в последнем неравенстве $u=\left(A_{\varepsilon}^{-1}-A^{-1}\right) v$, из (24) получим утверждение леммы.

2.3. Существование и свойства решений масштабированной задачи (20)-(23). Запишем задачу (20)-(23) в операторной форме в пространстве $H$ :

$$
\partial_{t} u+A_{\alpha, \varepsilon} u=B(u)+h_{\varepsilon}, \quad t>0, \quad u(0)=u_{0},
$$

где $h_{\varepsilon}=\left(h_{1}(x, \varepsilon y) ; h_{2}(x, \varepsilon y)\right) \in H_{\varepsilon}^{1}$ и отображение $B$ имеет вид

$$
(B(u))(x, y)=\left(f_{1}\left(u_{1}(x, y)\right) ; f_{2}\left(u_{2}(x, y)\right)\right) .
$$

Отображение $B$ обладает следуюшими свойствами. 
Лемма 2.2. Пусть выполнено (5). Тогда В отображает пространство $H^{1}=H^{1}\left(\Omega_{1}\right) \oplus H^{1}\left(\Omega_{2}\right)$ в $L^{2}(\Omega)$, причем

$$
\begin{gathered}
\|B(u)\| \leqslant c\left(1+\|u\|_{H^{1}}^{p+2}\right), \\
\|B(u)-B(v)\| \leqslant c\|u-v\|_{H^{1}}\left(1+\|u\|_{H^{1}}^{p+1}+\|v\|_{H^{1}}^{p+1}\right)
\end{gathered}
$$

для всех $и, v \in H^{1}$. Если рассматривать $B$ как отображсение из $H^{1}$ в двойственное пространство $H^{-1} \equiv\left[H^{1}\left(\Omega_{1}\right)\right]^{\prime} \oplus\left[H^{1}\left(\Omega_{2}\right)\right]^{\prime}$, то

$$
\|B(u)-B(v)\|_{H^{-1}} \leqslant c\|u-v\|\left(1+\|u\|_{H^{1}}^{p+1}+\|v\|_{H^{1}}^{p+1}\right), \quad u, v \in H^{1} .
$$

Кроме того, отображение $B: H^{1} \rightarrow L^{2}(\Omega)$ дифференцируемо по Фреше, его дифференциал задается формулой $B^{\prime}(u) v=\left(f_{1}^{\prime}\left(u_{1}\right) v_{1} ; f_{2}^{\prime}\left(u_{2}\right) v_{2}\right)$ для $u, v \in H^{1}$. При этом для всех $u, v \in H^{1}$ справедливы оценки

$$
\begin{gathered}
\left\|B^{\prime}(u)\right\|_{\mathscr{L}\left(H^{1} ; H\right)} \leqslant c\left(1+\|u\|_{H^{1}}^{p+1}\right), \\
\left\|B^{\prime}(u)-B^{\prime}(v)\right\|_{\mathscr{L}\left(H^{1} ; H\right)} \leqslant c\|u-v\|_{H^{1}}\left(1+\|u\|_{H^{1}}^{p}+\|v\|_{H^{1}}^{p}\right) .
\end{gathered}
$$

Если рассматривать $B^{\prime}$ как отображение из $H^{1}$ в $\mathscr{L}\left(H^{1} ; H^{-1}\right)$, то

$$
\left\|B^{\prime}(u)-B^{\prime}(v)\right\|_{\mathscr{L}\left(H^{1} ; H^{-1}\right)} \leqslant c\|u-v\|\left(1+\|u\|_{H^{1}}^{p}+\|v\|_{H^{1}}^{p}\right)
$$

для всех $u, v \in H^{1}$.

ДоКАЗАТЕЛЬСТво носит стандартный характер и опирается на непрерывность вложения $H^{s}\left(\Omega_{i}\right)$ в $L^{\theta}\left(\Omega_{i}\right)$ при $(d+1) / \theta \geqslant(d+1) / 2-s, s \geqslant 0, \theta \geqslant 2$.

ОПРЕДЕЛЕНИЕ 2.1. Будем говорить, что функция $u(t):[0,+\infty) \rightarrow H$ является сильныц решением задачи (30), если $u(0)=u_{0}$,

$$
u(t) \in C\left([0,+\infty) ; H_{\varepsilon}^{1}\right) \cap C^{1}\left((0,+\infty) ; H_{\varepsilon}^{1}\right) \cap C\left((0,+\infty) ; \mathrm{D}\left(A_{\alpha, \varepsilon}\right)\right)
$$

и $u(t)$ удовлетворяет уравнению (30) при всех $t \in(0,+\infty)$.

ТеОРема 2.1. Пусть выполнены условия (5)-(9). Тогда для всех $\alpha \in \mathbb{R}$, $0<\varepsilon \leqslant 1$ и для любого $u_{0} \in H_{\varepsilon}^{1}$ задача Коши (30) имеет единственное сильное решение $u_{\alpha, \varepsilon}(t)$. При этом для любого $r>0$

$$
\begin{gathered}
\sup _{t \geqslant 0}\left[\sup _{a_{\alpha, \varepsilon}(u, u) \leqslant r} a_{\alpha, \varepsilon}\left(S_{\alpha, \varepsilon}(t) u, S_{\alpha, \varepsilon}(t) u\right)\right] \leqslant c(r) \\
t^{1 / 2} \sup \left\{\left\|A_{\alpha, \varepsilon} S_{\alpha, \varepsilon}(t) u\right\|: u \in H_{\varepsilon}^{1}, a_{\alpha, \varepsilon}(u, u) \leqslant r\right\} \leqslant c(r)(1+t)^{1 / 2}, \quad t>0
\end{gathered}
$$

где $S_{\alpha, \varepsilon}(t)$ - эволючионная полугруппа, порождаемая в пространстве $H_{\varepsilon}^{1}$ задачей $(30), S_{\alpha, \varepsilon}(t) u_{0} \equiv u_{\alpha, \varepsilon}(t)$.

Кроме того, существует такое число $R>0$, что для любого шара $U_{\varepsilon, r}=$ $\left\{u \in H_{\varepsilon}^{1}: a_{\alpha, \varepsilon}(u, u) \leqslant r\right\}$ найдется такое $t=t(r)$, что

$$
\sup _{u \in U_{\varepsilon, r}} a_{\alpha, \varepsilon}\left(S_{\alpha, \varepsilon}(t) u, S_{\alpha, \varepsilon}(t) u\right) \leqslant R^{2} \quad n p u \quad t \geqslant t(r), \quad \varepsilon \in(0,1],
$$

m.е. полугруппа $S_{\alpha, \varepsilon}(t)$ диссипативна в $H_{\varepsilon}^{1}$ равномерно по $\varepsilon$. 
ДоказАТЕЛЬство. Сушествование и единственность сильных решений устанавливается стандартным методом (см., например, [5], [13] или [15]). Что касается равномерных оценок (31)-(33), то они могут быть получены с помощью хорошо известных подходов (см., например, [5]), развитых для нелинейных параболических задач. В случае сингулярно возмущенных задач эти подходы ранее применялись, например, в [1], [9], [10].

Опишем схему рассуждений применительно к рассматриваемой задаче. Прежде всего заметим, что для любого сильного решения $u_{\alpha, \varepsilon}(t)$ при $t>0$ справедливы соотношения

$$
\frac{1}{2} \partial_{t}\left\|u_{\alpha, \varepsilon}(t)\right\|^{2}+a_{\alpha, \varepsilon}\left(u_{\alpha, \varepsilon}(t), u_{\alpha, \varepsilon}(t)\right)=\left(B\left(u_{\alpha, \varepsilon}(t)\right)+h_{\varepsilon}, u_{\alpha, \varepsilon}(t)\right)_{H}
$$

и

$$
\partial_{t} \varphi_{\alpha, \varepsilon}\left(u_{\alpha, \varepsilon}(t)\right)+\left\|\partial_{t} u_{\alpha, \varepsilon}(t)\right\|^{2}=0
$$

где

$$
\varphi_{\alpha, \varepsilon}(u)=\frac{1}{2} a_{\alpha, \varepsilon}(u, u)-\sum_{i=1}^{2} \int_{\Omega_{i}} F_{i}\left(u_{i}\right) d x d y-\int_{\Omega} u h_{\varepsilon} d x d y .
$$

Здесь $F_{i}(u)=\int_{0}^{u} f_{i}(\xi) d \xi$. Используя $(5),(6)$ и (8), легко обнаружить, что сушествуют положительные константы $c_{0}, c_{1}$ и $K$ такие, что

$$
c_{0} a_{\alpha, \varepsilon}(u, u) \leqslant \frac{1}{2}\|u\|^{2}+\varphi_{\alpha, \varepsilon}(u)+K \leqslant c_{1}\left(1+a_{\alpha, \varepsilon}(u, u)\right)^{(p+3) / 2} .
$$

Пусть $V_{\alpha, \varepsilon}(u)=\frac{1}{2}\|u\|^{2}+\varphi_{\alpha, \varepsilon}(u)+K$. Тогда из (6), (7), (34) и (35) вытекает, что существуют константы $\omega>0$ и $C>0$ такие, что

$$
\partial_{t} V_{\alpha, \varepsilon}\left(u_{\alpha, \varepsilon}(t)\right)+\omega V_{\alpha, \varepsilon}\left(u_{\alpha, \varepsilon}(t)\right) \leqslant C .
$$

Отсюда после интегрирования с помощью (37) получаем соотношения (31) и (33).

Для доказательства (32) прежде всего отметим, что при условии $a_{\alpha, \varepsilon}\left(u_{0}, u_{0}\right) \leqslant$ $r$ из (35) и (37) вытекает оценка

$$
\int_{0}^{t}\left\|\partial_{t} u_{\alpha, \varepsilon}(\tau)\right\|^{2} d \tau \leqslant c(r), \quad t>0 .
$$

Дальнейшие рассуждения носят формальный характер. Их можно сделать строгими, рассматривая соответствующие галёркинские аппроксимации.

Пусть $v_{\alpha, \varepsilon}(t)=\partial_{t} u_{\alpha, \varepsilon}(t)$. Тогда функция $v_{\alpha, \varepsilon}(t)$ удовлетворяет уравнению

$$
\partial_{t} v_{\alpha, \varepsilon}(t)+A_{\alpha, \varepsilon} v_{\alpha, \varepsilon}(t)=B^{\prime}\left(u_{\alpha, \varepsilon}(t)\right) v_{\alpha, \varepsilon}(t) .
$$

В силу леммы 2.2

$$
\left(B^{\prime}(u) v, v\right)_{H} \leqslant \frac{1}{2} a_{\alpha, \varepsilon}(v, v)+C\|v\|^{2}\left(1+a_{\alpha, \varepsilon}(u, u)\right)^{p+1} .
$$

Поэтому из (31) и (39) получаем, что

$$
\partial_{t}\left\|v_{\alpha, \varepsilon}(t)\right\|^{2}+a_{\alpha, \varepsilon}\left(v_{\alpha, \varepsilon}(t), v_{\alpha, \varepsilon}(t)\right) \leqslant c(r)\left\|\partial_{t} u_{\alpha, \varepsilon}(t)\right\|^{2}, \quad t>0
$$

при условии $a_{\alpha, \varepsilon}\left(u_{0}, u_{0}\right) \leqslant r$. Отсюда и из $(38)$ вытекает, что

$$
t\left\|\partial_{t} u_{\alpha, \varepsilon}(t)\right\|^{2}+\int_{0}^{t} \tau a_{\alpha, \varepsilon}\left(\partial_{t} u_{\alpha, \varepsilon}(\tau), \partial_{t} u_{\alpha, \varepsilon}(\tau)\right) d \tau \leqslant c(r)(1+t) .
$$

Поэтому, используя (30) и лемму 2.2 , легко получить (32). 
ЗАмечание 2.1. Используя соотношение (31), а также лемму 2.2 и рассуждения, аналогичные приведенньм вьше, нетрудно получить равномерную оценку

$$
\left\|S_{\varepsilon}(t) u^{1}-S_{\varepsilon}(t) u^{2}\right\|_{1, \varepsilon} \leqslant C e^{K t}\left\|u^{1}-u^{2}\right\|_{1, \varepsilon}, \quad t \geqslant 0
$$

для любых $u^{i} \in H_{\varepsilon}^{1}$ таких, что $a_{\alpha, \varepsilon}\left(u^{i}, u^{i}\right) \leqslant r$, где $r>0, \varepsilon \in(0,1]$, а числа $C$, $K>0$ зависят только от $r$.

Из теоремы 2.1 и стандартных результатов о существовании и свойствах глобальных аттракторов (см., например, [5], [6], [13]) вытекает следующее утверждение.

СлЕДСТВИЕ 2.1. Для каждой пары $(\alpha, \varepsilon) \in \mathbb{R} \times(0,1]$ задача $(30)$ порождает компактную диссипативную систему $\left(H_{\varepsilon}^{1}, S_{\alpha, \varepsilon}(t)\right)$, обладающую компактнымм глобальным аттрактором $\mathscr{A}_{\alpha, \varepsilon}$, лежащим в пространстве $H^{2}\left(\Omega_{1}\right) \oplus$ $H^{2}\left(\Omega_{2}\right)$. Этот аттрактор имеет вид $\mathscr{A}_{\alpha, \varepsilon}=W^{u}\left(\mathscr{N}_{\alpha, \varepsilon}\right)$, где $W^{u}\left(\mathscr{N}_{\alpha, \varepsilon}\right)-$ неустойчивое многообразие, выходящее из множества $\mathscr{N}_{\alpha, \varepsilon}$ неподвижных точек задачи (30),

$$
\mathscr{N}_{\alpha, \varepsilon}=\left\{u \in \mathrm{D}\left(A_{\alpha, \varepsilon}\right): A_{\alpha, \varepsilon} u=B(u)\right\} .
$$

При этом полнъе траектории $\left\{u_{\alpha, \varepsilon}(t), t \in \mathbb{R}\right\}$, лежащие в аттракторе, обладают следующими свойствами:

$$
\begin{gathered}
\sup _{t \in \mathbb{R}}\left\{a_{\alpha, \varepsilon}\left(u_{\alpha, \varepsilon}(t), u_{\alpha, \varepsilon}(t)\right)+\left\|A_{\alpha, \varepsilon} u_{\alpha, \varepsilon}(t)\right\|^{2}\right\} \leqslant R^{2}, \\
\sup _{t \in \mathbb{R}}\left\{\left\|\partial_{t} u_{\alpha, \varepsilon}(t)\right\|^{2}+\int_{t}^{t+1} a_{\alpha, \varepsilon}\left(\partial_{t} u_{\alpha, \varepsilon}(\tau), \partial_{t} u_{\alpha, \varepsilon}(\tau)\right) d \tau\right\} \leqslant R^{2},
\end{gathered}
$$

әде константа $R$ не зависит от $\varepsilon$.

ЗАмечание 2.2. Очевидно, что эволюционные операторы $T_{\alpha, \varepsilon}(t)$ и $S_{\alpha, \varepsilon}(t)$ связаны соотношением $T_{\alpha, \varepsilon}(t)=R_{\varepsilon}^{-1} S_{\alpha, \varepsilon}(t) R_{\varepsilon}$, где оператор растяжения $R_{\varepsilon}$ действует из $H^{1}\left(\Omega_{1, \varepsilon}\right) \oplus H^{1}\left(\Omega_{2, \varepsilon}\right)$ в $H^{1}\left(\Omega_{1}\right) \oplus H^{1}\left(\Omega_{2}\right)$ по формуле

$$
\left[R_{\varepsilon} u\right](x, y)=u(x, \varepsilon y), \quad x \in \Gamma, \quad y \in(-1,1) .
$$

Это наблюдение, в частности, позволяет из следствия 2.1 извлечь утверждение теоремы 1.1 об аттракторе задачи (1)-(4).

\section{§3. Сходимость эволюционных операторов}

В этом параграфе изучается поведение при $\varepsilon \rightarrow 0$ решений масштабированной задачи (20)-(23) на конечных интервалах времени. Установленные здесь утверждения используются при изучении асимптотического поведения аттрактора $\mathscr{A}_{\alpha, \varepsilon}$. Их следствием являются также теоремы 1.2 и 1.3.

ТЕОрема 3.1. Предположим, что $\alpha \geqslant 1$. Пусть $и(t)=u_{\varepsilon}(t)-$ решение задачи (20)-(23) $с\left\|u_{0}\right\|_{1, \varepsilon} \leqslant r, \varepsilon \in(0,1], v(t)$ - решение задачи (10) при $\alpha=1$ 
$u v(t)$ - решение задачи (11) при $\alpha>1$ с начальным условием $v(0)=M u(0)$. Обозначим $h(\varepsilon)=\|h\|_{\mathscr{F}_{\varepsilon}}^{2} \equiv\left\|h_{1}\right\|_{H^{1}\left(\Omega_{1, \varepsilon}\right)}^{2}+\left\|h_{2}\right\|_{H^{1}\left(\Omega_{2, \varepsilon}\right)}^{2}$. Тогда для каждого $r>0$ существуют константы $c_{1}(r)$ и $c_{2}(r)$ такие, что

$$
\begin{gathered}
\|M u(t)-v(t)\|_{\mathbf{L}^{2}}^{2}+\int_{0}^{t}\|M u(\tau)-v(\tau)\|_{\mathscr{F}_{0}}^{2} d \tau \leqslant c_{1}(r)\left(\varepsilon^{4}+\varepsilon h(\varepsilon)\right) e^{c_{2}(r) t} \\
\|u(t)-v(t)\|^{2}+\int_{0}^{t}\|u(\tau)-v(\tau)\|_{1, \varepsilon}^{2} d \tau \leqslant c_{1}(r)\left(\varepsilon^{2}+\varepsilon h(\varepsilon)\right) e^{c_{2}(r) t} \\
t^{1 / 2}\|u(t)-v(t)\|_{1, \varepsilon}^{2} \leqslant c_{1}(r)\left(\varepsilon^{2}+\varepsilon h(\varepsilon)\right)^{1 / 2} e^{c_{2}(r) t}
\end{gathered}
$$

ДокАЗАТЕльство. Рассуждения проведем только для (наиболее сложного) случая $\alpha=1$. При $\alpha>1$ рассуждения аналогичны.

Из (20) вытекает, что величины $M_{i} u_{i}, i=1,2$, удовлетворяют уравнению

$\partial_{t} M_{i} u_{i}-\nu_{i} \Delta_{\varepsilon} M_{i} u_{i}-(-1)^{i} k(x)\left(u_{1}(x, 0)-u_{2}(x, 0)\right)+a M_{i} u_{i}=M_{i}\left(f_{i}\left(u_{i}\right)+h_{i}^{\varepsilon}\right)$,

откуда с учетом (20) легко получить соотношение

$$
\left(\partial_{t} n_{i}, n_{i}\right)_{L^{2}\left(\Omega_{i}\right)}-\nu_{i}\left(\Delta_{\varepsilon} n_{i}, n_{i}\right)_{L^{2}\left(\Omega_{i}\right)}+a\left(n_{i}, n_{i}\right)_{L^{2}\left(\Omega_{i}\right)}=\left(f_{i}\left(u_{i}\right)+h_{i}^{\varepsilon}, n_{i}\right)_{L^{2}\left(\Omega_{i}\right)},
$$

где $n_{i}=u_{i}(t)-M_{i} u_{i}(t)$. Следовательно,

$$
\begin{aligned}
\frac{1}{2} \partial_{t}\left\|n_{i}\right\|_{L^{2}\left(\Omega_{i}\right)}^{2}+\nu_{i}\left\|\nabla_{\varepsilon} n_{i}\right\|_{L^{2}\left(\Omega_{i}\right)}^{2}+a\left\|n_{i}\right\|_{L^{2}\left(\Omega_{i}\right)}^{2} \\
=(-1)^{i} \int_{\Gamma} k(x)\left(u_{1}(x, 0)-u_{2}(x, 0)\right)\left(u_{i}(x, 0)-M_{i} u_{i}\right) d x \\
\quad+\left(f_{i}\left(u_{i}\right), n_{i}\right)_{L^{2}\left(\Omega_{i}\right)}+\left(h_{i}^{\varepsilon}, n_{i}\right)_{L^{2}\left(\Omega_{i}\right)} .
\end{aligned}
$$

Используя (31) и (26), имеем, что

$$
\left|\int_{\Gamma}\right| \leqslant c(r) \varepsilon^{2}+\frac{\nu_{i}}{4}\left\|\nabla_{\varepsilon} n_{i}\right\|_{L^{2}\left(\Omega_{i}\right)}^{2}
$$

В силу леммы 2.2 , соотношения (31) и оценки (25) получаем, что

$$
\left|\left(f_{i}\left(u_{i}\right), n_{i}\right)_{L^{2}\left(\Omega_{i}\right)}\right| \leqslant c(r) \varepsilon^{2}+\frac{\nu_{i}}{4}\left\|\nabla_{\varepsilon} n_{i}\right\|_{L^{2}\left(\Omega_{i}\right)}^{2} .
$$

Аналогично находим, что

$$
\left|\left(h_{i}^{\varepsilon}, n_{i}\right)_{L^{2}\left(\Omega_{i}\right)}\right| \leqslant c \varepsilon^{2}\left\|\left(I-M_{i}\right) h_{i}^{\varepsilon}\right\|_{L^{2}\left(\Omega_{i}\right)}^{2}+\frac{\nu_{i}}{4}\left\|\nabla_{\varepsilon} n_{i}\right\|_{L^{2}\left(\Omega_{i}\right)}^{2} .
$$

Очевидно, что норма $\left\|\left(I-M_{i}\right) h_{i}^{\varepsilon}\right\|_{L^{2}\left(\Omega_{i}\right)}^{2}$ равномерно ограничена. Поэтому из (50)-(52) выводим, что

$$
\partial_{t}\|n(t)\|^{2}+c\|n(t)\|_{1, \varepsilon}^{2} \leqslant c(r) \varepsilon^{2} .
$$


Так как из (25) вытекает, что $\varepsilon^{-2}\|n\|^{2} \leqslant\|n(t)\|_{1, \varepsilon}^{2}$, то после интегрирования и применения леммы Гронуолла получаются оценки

$$
\|n(t)\|^{2} \leqslant c_{1}(r) \varepsilon^{2} e^{-c_{0} t \varepsilon^{-2}}+c_{2}(r) \varepsilon^{4}, \quad t \geqslant 0,
$$

и

$$
\int_{0}^{t}\|n(s)\|_{1, \varepsilon}^{2} d s \leqslant c(r)(1+t) \varepsilon^{2}, \quad t \geqslant 0
$$

где $n(t)=N u(t)$. Далее, из (10) и (48) получаем

$$
\begin{aligned}
& \frac{1}{2} \partial_{t}\left\|M_{i} u_{i}-v_{i}\right\|_{L^{2}\left(\Omega_{i}\right)}^{2}+\nu_{i}\left\|\nabla\left(M_{i} u_{i}-v_{i}\right)\right\|_{L^{2}\left(\Omega_{i}\right)}^{2}+a\left\|M_{i} u_{i}-v_{i}\right\|_{L^{2}\left(\Omega_{i}\right)}^{2} \\
& \leqslant \int_{\Gamma}\left|k(x)\left(\left(u_{1}(x, 0)-v_{1}\right)-\left(u_{2}(x, 0)-v_{2}\right)\right)\left(M_{i} u_{i}-v_{i}\right)\right| d x \\
& \quad+\left|\left(f_{i}\left(u_{i}\right)-f_{i}\left(v_{i}\right), M_{i} u_{i}-v_{i}\right)_{L^{2}\left(\Omega_{i}\right)}\right|+\left|\left(M_{i} h^{\varepsilon}-\gamma_{i} h, M_{i} u_{i}-v_{i}\right)_{L^{2}\left(\Omega_{i}\right)}\right|
\end{aligned}
$$

где $\gamma_{i} h=h_{i}(x, 0)$. Используя $(25)$, легко обнаружить, что

$$
\int_{\Gamma}|\cdot| \leqslant c\left(\|m-v\|_{L^{2}(\Omega)}^{2}+\varepsilon^{2}\|n\|_{1, \varepsilon}^{2}\right)
$$

где $m(t)=M u(t)$. Из леммы 2.2 и оценки $(31)$ вытекает, что

$$
\begin{aligned}
\left|(B(u)-B(v), m-v)_{H}\right| & \leqslant c(r)\|u-v\|\|m-v\|_{H^{1}} \\
& \leqslant \delta\|m-v\|_{H^{1}}^{2}+\delta^{-1} c(r)\left(\|m-v\|^{2}+\varepsilon^{2}\|n\|_{1, \varepsilon}^{2}\right)
\end{aligned}
$$

для любого $\delta>0$. Выбирая в (57) достаточно малое $\delta$ и используя (56), а также очевидное неравенство

$$
\sum_{i=1,2} \int_{\Gamma}\left|M_{i} h^{\varepsilon}-\gamma_{i} h\right|^{2} d x \leqslant \varepsilon \sum_{i=1,2}\left\|h_{i}\right\|_{H^{1}\left(\Omega_{i, \varepsilon}\right)}^{2} \equiv \varepsilon h(\varepsilon)
$$

из (55) выводим, что

$$
\partial_{t}\|m-v\|^{2}+c_{1}\|m-v\|_{1, \varepsilon}^{2} \leqslant c(r)\left(\|m-v\|^{2}+\varepsilon^{2}\|n\|_{1, \varepsilon}^{2}\right)+c_{2} \varepsilon h(\varepsilon) .
$$

Интегрируя последнее неравенство от 0 до $t$, применяя лемму Гронуолла, оценку $(54)$ и равенство $m(0)=v(0)$, получаем неравенство (45).

Оценка (46) вытекает из (53), (54) и (45).

Докажем теперь неравенство (47). Прежде всего заметим, что для $u \in \mathrm{D}\left(A_{\varepsilon}\right)$ и $v \in \mathrm{D}(A)$ справедливо неравенство

$$
\|u-v\|_{1, \varepsilon}^{2} \leqslant C_{1}\|u-v\|\left(\left\|A_{\varepsilon} u\right\|+\|A v\|\right)+C_{2} \varepsilon^{2}\|u\|^{2} .
$$


Действительно, из определения нормы $\|\cdot\|_{1, \varepsilon}$ после интегрирования по частям, используя граничное условие (23), для $w=u-v$ имеем, что

$$
\|w\|_{1, \varepsilon}^{2} \leqslant\left(A_{\varepsilon} u-A v, w\right)_{H}+C \sum_{i, j=1,2}\left\|u_{i}\right\|_{L^{2}(\Gamma)}\left\|w_{j}\right\|_{L^{2}(\Gamma)} .
$$

Так как

$$
\left\|u_{i}\right\|_{L^{2}(\Gamma)} \leqslant\left\|u_{i}\right\|_{L^{2}\left(\Omega_{i}\right)}^{2}+2\left\|u_{i}\right\|_{L^{2}\left(\Omega_{i}\right)}\left\|\partial_{y} u_{i}\right\|_{L^{2}\left(\Omega_{i}\right)}
$$

и $\partial_{y} u_{i}=\partial_{y} w_{i}$, то нетрудно обнаружить, что

$$
\left\|u_{i}\right\|_{L^{2}(\Gamma)}\left\|w_{j}\right\|_{L^{2}(\Gamma)} \leqslant C_{1}\|w\|(\|u\|+\|w\|)+C_{2} \varepsilon^{2}\|u\|^{2}+\frac{1}{2 \varepsilon^{2}} \sum_{i=1,2}\left\|\partial_{y} w_{i}\right\|_{L^{2}\left(\Omega_{i}\right)}^{2} .
$$

Отсюда и из (59) вытекает (58).

Используя (31) и (32) вместе с аналогичным соотношением для предельной задачи, из (58) получаем, что

$$
t^{1 / 2}\|u(t)-v(t)\|_{1, \varepsilon}^{2} \leqslant c_{1}(r)(1+t)^{1 / 2}\|u(t)-v(t)\|+c_{2}(r) \varepsilon^{2} t^{1 / 2} .
$$

Отсюда и из (46) вытекает оценка (47).

ДОКАЗАТЕЛЬСТво теОРемЫ 1.2. Очевидно, что

$$
M^{\varepsilon} T_{\alpha, \varepsilon} w_{0}=M S_{\alpha, \varepsilon} R_{\varepsilon} w_{0}, \quad M^{\varepsilon} w_{0}=M R_{\varepsilon} w_{0}
$$

где оператор $R_{\varepsilon}$ определен равенством (44). Кроме того,

$$
\left\|R_{\varepsilon} w_{0}\right\|_{1, \varepsilon}^{2} \leqslant \frac{c}{\varepsilon}\left(\left\|w_{1,0}\right\|_{H^{1}\left(\Omega_{1, \varepsilon}\right)}^{2}+\left\|w_{2,0}\right\|_{H^{1}\left(\Omega_{2, \varepsilon}\right)}^{2}\right)
$$

Поэтому (13) вытекает из неравенства (45). Что касается (14), то следует воспользоваться соотношением (13), интерполяционньм неравенством $\|u\|_{H^{s}(\Gamma)} \leqslant$ $C\|u\|_{L^{2}(\Gamma)}^{1-s}\|u\|_{H^{1}(\Gamma)}^{s}$ и равномерной оценкой $(31)$.

Рассмотрим теперь случай $\alpha<1$.


с начальныци условиями $u_{1,0}(x, y)=v_{0}(x)$ u $u_{2,0}(x, y)=v_{0}(x)$, где $v_{0} \in H^{1}(\Gamma)$. Тогда

$$
\lim _{\varepsilon \rightarrow 0} \sum_{i=1,2} \sup _{t \in[0, T]}\left\|M u_{i}^{\varepsilon}(t)-v(t)\right\|_{H^{s}(\Gamma)}=0
$$

для любого $0 \leqslant s<1$, где $v(t)-$ решение задачи (12). 
ДоказАТЕЛЬСтво. Очевидно, что $a_{\alpha, \varepsilon}\left(u_{0}, u_{0}\right)$ не зависит от $\varepsilon$. Поэтому из теоремы 2.1 вытекает равномерная оценка

$$
\sum_{i=1,2}\left(\left\|u_{i}^{\varepsilon}(t)\right\|_{H^{1}\left(\Omega_{i}\right)}^{2}+\frac{1}{\varepsilon^{2}}\left\|\partial_{y} u_{i}^{\varepsilon}(t)\right\|_{L^{2}\left(\Omega_{i}\right)}^{2}\right)+\frac{1}{\varepsilon^{1-\alpha}} \int_{\Gamma}\left|u_{1}^{\varepsilon}(t)-u_{2}^{\varepsilon}(t)\right|^{2} d x \leqslant C
$$

для всех $t \in[0, T]$. Кроме того, из (38) имеем, что

$$
\sum_{i=1,2} \int_{0}^{T}\left\|\partial_{t} u_{i}^{\varepsilon}(t)\right\|_{L^{2}\left(\Omega_{i}\right)}^{2} d t \leqslant C
$$

Поэтому из теоремы Дубинского [5; гл. I, $\S 1$, теорема 4] вытекает, что сушествуют функция

$$
v(t) \in C\left(0, T ; L^{2}(\Gamma)\right) \cap L^{\infty}\left(0, T ; H^{1}(\Gamma)\right)
$$

и последовательность $\left\{\varepsilon_{n}\right\}$ такие, что

$$
\lim _{n \rightarrow \infty} \sum_{i=1,2} \sup _{t \in[0, T]}\left\|u_{i}^{\varepsilon_{n}}(t)-v(t)\right\|_{L^{2}\left(\Omega_{i}\right)}=0
$$

и $u_{i}^{\varepsilon_{n}}(t) \rightarrow v(t) *$-слабо в $L^{\infty}\left(0, T ; H^{1}\left(\Omega_{i}\right)\right)$. Покажем, что $v(t)$ является решением задачи (12). Для этого воспользуемся вариационной формой задачи (20)-(23):

$$
\left(u^{\varepsilon}(t), \psi\right)_{H}+\int_{0}^{t} a_{\alpha, \varepsilon}\left(u^{\varepsilon}(\tau), \psi\right) d \tau=\left(u_{0}, \psi\right)_{H}+\int_{0}^{t}\left(B\left(u^{\varepsilon}(\tau)\right)+h^{\varepsilon}, \psi\right)_{H} d \tau
$$

для любых $\psi \in H_{\varepsilon}^{1}$. Выбирая функцию $\psi$ в виде $\psi=\left(\psi_{0}(x) ; \psi_{0}(x)\right)$, где $\psi_{0}(x) \in$ $H^{1}(\Gamma)$, находим, что

$$
a_{\alpha, \varepsilon}\left(u^{\varepsilon}(\tau), \psi\right)=\sum_{i=1,2} \int_{\Omega_{i}}\left(\nu_{i} \nabla_{x} u_{i}^{\varepsilon}(\tau, x, y) \cdot \nabla_{x} \psi_{0}(x)+a u_{i}^{\varepsilon}(\tau, x, y) \psi_{0}(x)\right) d x d y
$$

Поэтому, используя лемму 2.2 , можно совершить в (63) предельньй переход и убедиться в том, что $v(t)$ является решением задачи (12).

Неравенство (61) вытекает из (62) и равномерной оценки (31).

ДоКАЗАТЕЛЬСТво ТЕОРемЫ 1.3. Достаточно воспользоваться равенством $M^{\varepsilon} w^{\varepsilon}=M u^{\varepsilon}$ и теоремой 3.2 . 


\section{§4. Асимптотические свойства аттрактора масштабированной системы}

В этом параграфе устанавливаются асимптотические свойства аттрактора $\mathscr{A}_{\alpha, \varepsilon}$ масштабированной задачи (20)-(23), позволяющие доказать теоремы 1.4-1.6.

\section{1. Полунепрерывность сверху.}

Теорема 4.1. Для любых $\alpha \geqslant 1$ справедливо соотношение

$$
\lim _{\varepsilon \rightarrow 0} \sup _{u \in \mathscr{A}_{\alpha, \varepsilon}} \inf _{v \in \mathscr{A}_{\alpha}}\|u-v\|_{1, \varepsilon}=0
$$

где $\mathscr{A}_{\alpha}$ - глобальный аттрактор задачи (10) при $\alpha=1$ u (11) при $\alpha>1$ соответственно.

ДоКАЗАТЕЛЬСтво. Воспользуемся соответствуюшим рассуждением из [1] (см. также [5]). Пусть $u \in \mathscr{A}_{\alpha, \varepsilon}$. Тогда в силу (42)

$$
\|M u\|_{H^{1}(\Gamma)} \leqslant\|u\|_{1, \varepsilon} \leqslant R, \quad \varepsilon \in(0,1]
$$

для некоторого $R>0$. Поэтому для любого $\delta>0$ существует такое $T=$ $T(\delta, R)>0$, что

$$
\inf _{w \in \mathscr{A}_{\alpha}}\left\|S_{\alpha}(t) M u-w\right\|_{H^{1}(\Gamma)} \leqslant \delta, \quad u \in \mathscr{A}_{\alpha, \varepsilon}, \quad t \geqslant T
$$

Из (47) следует, что для $0<\tau \leqslant t \leqslant T$

$$
\left\|S_{\alpha, \varepsilon}(t) u-S_{\alpha}(t) M u\right\|_{1, \varepsilon} \leqslant \tau^{-1 / 4}\left(\varepsilon^{2}+\varepsilon h(\varepsilon)\right)^{1 / 4} c_{1}(R) e^{c_{2}(R) T}
$$

при всех $u \in \mathscr{A}_{\alpha, \varepsilon}$. Соотношения (65) и (66) влекут оценку

$$
\inf _{w \in \mathscr{A}_{\alpha}}\left\|S_{\alpha, \varepsilon}(T) u-w\right\|_{1, \varepsilon} \leqslant \delta+\tau^{-1 / 4}\left(\varepsilon^{2}+\varepsilon h(\varepsilon)\right)^{1 / 4} c_{1}(R) e^{c_{2}(R) T}
$$

для всех $u \in \mathscr{A}_{\alpha, \varepsilon}$. Теперь утверждение теоремы сразу следует из последнего неравенства, так как $S_{\alpha, \varepsilon}(T) \mathscr{A}_{\alpha, \varepsilon}=\mathscr{A}_{\alpha, \varepsilon}, \varepsilon \in(0,1]$.

ДоКАЗАТЕЛЬСТво ТЕОРЕМЫ 1.4. Так как $\left\|M_{i} f_{i}\right\|_{L^{2}(\Gamma)} \leqslant\left\|f_{i}\right\|_{L^{2}\left(\Omega_{i}\right)}$ для всех $f_{i} \in L^{2}\left(\Omega_{i}\right)$, то

$$
\|M u-v\|_{H^{1}(\Gamma) \oplus H^{1}(\Gamma)}=\|M(u-v)\|_{H^{1}(\Gamma) \oplus H^{1}(\Gamma)} \leqslant c\|u-v\|_{1, \varepsilon} .
$$

Поэтому утверждение теоремы 1.4 вытекает из теоремы 4.1.

Точно так же для доказательства теоремы 1.5 достаточно установить следующее утверждение. 
Tеорема 4.2. Пусть $\alpha<1$ и $\mathscr{A}_{\alpha}$ определяется формулой

$$
\mathscr{A}_{\alpha}=\left\{(v ; v) \in H^{1}(\Gamma) \oplus H^{1}(\Gamma): v \in \widetilde{\mathscr{A}}\right\},
$$

где $\tilde{\mathscr{A}}$ - глобальный аттрактор задачи (12). Тогда имеет место соотношение (64).

ДокАЗАТЕЛЬСтво. Предположим, что (64) неверно. Тогда существуют $\eta>0$ и последовательности $\varepsilon_{n} \rightarrow 0$ и $u^{n} \in \mathscr{A}_{\alpha, \varepsilon_{n}}$ такие, что

$$
\inf _{v \in \mathscr{A}_{\alpha}}\left\|u^{n}-v\right\|_{1, \varepsilon_{n}}>\eta
$$

Пусть $\left\{u^{n}(t): t \in \mathbb{R}\right\}$ - полная траектория, лежащая в аттракторе $\mathscr{A}_{\alpha, \varepsilon_{n}}$ и проходящая через $u^{n}, u^{n}(0)=u^{n}$. Следствие 2.1 дает, что

$$
\begin{gathered}
\sum_{i=1}^{2}\left(\left\|\nabla_{x} u_{i}^{n}(t)\right\|_{L^{2}\left(\Omega_{i}\right)}^{2}+\frac{1}{\varepsilon_{n}^{2}}\left\|\partial_{y} u_{i}^{n}(t)\right\|_{L^{2}\left(\Omega_{i}\right)}^{2}+\left\|u_{i}^{n}(t)\right\|_{L^{2}\left(\Omega_{i}\right)}^{2}\right) \leqslant C \\
\frac{1}{\varepsilon^{1-\alpha}} \int_{\Gamma}\left|u_{1}^{n}(t)-u_{2}^{n}(t)\right|^{2} d x \leqslant C, \quad \int_{t}^{t+1}\left\|\partial_{t} u_{i}^{n}(\tau)\right\|_{1, \varepsilon_{n}}^{2} d \tau \leqslant C
\end{gathered}
$$

для всех $t \in \mathbb{R}$. В частности,

$$
\sup _{t \in \mathbb{R}}\left\{\left\|\partial_{y} u_{1}^{n}(t)\right\|_{L^{2}\left(\Omega_{1}\right)}^{2}+\left\|\partial_{y} u_{2}^{n}(t)\right\|_{L^{2}\left(\Omega_{2}\right)}^{2}+\int_{\Gamma}\left|u_{1}^{n}(t)-u_{2}^{n}(t)\right|^{2} d x\right\} \rightarrow 0
$$

при $n \rightarrow \infty$. Используя теорему Дубинского [5; гл. I, $\S 1$, теорема 4], заключаем, что существуют функция $v(t) \in C_{b}\left(\mathbb{R}, L^{2}(\Gamma)\right) \cap L^{\infty}\left(\mathbb{R}, H^{1}(\Gamma)\right)$ и подпоследовательность $\left\{n_{k}\right\}$ такие, что

$$
\max _{t \in[a, b]}\left\{\left\|u_{1}^{n_{k}}(t)-v(t)\right\|_{L^{2}\left(\Omega_{1}\right)}^{2}+\left\|u_{2}^{n_{k}}(t)-v(t)\right\|_{L^{2}\left(\Omega_{2}\right)}^{2}\right\} \rightarrow 0, \quad k \rightarrow \infty,
$$

и $u_{i}^{n_{k}}(t) \rightarrow v(t) *$-слабов $L^{\infty}\left(a, b ; H^{1}(\Gamma)\right)$ для любого интервала $[a, b] \subset \mathbb{R}$. Далее, как и при доказательстве теоремы 3.2 , переходя к пределу в вариационной формулировке задачи (20)-(23), нетрудно обнаружить, что $v(t)$ - ограниченное на $\mathbb{R}$ решение задачи (12) и $v(t) \in C_{b}\left(\mathbb{R}, H^{1}(\Gamma)\right)$. Поэтому оно принадлежит аттрактору $\widetilde{\mathscr{A}}$ и, значит, $\widetilde{v}(t) \equiv(v(t) ; v(t)) \in \mathscr{A}_{\alpha}$. При этом очевидно, что $\widetilde{v}(t) \in \mathrm{D}\left(A_{\alpha, \varepsilon}\right)$ и $\left\|A_{\alpha, \varepsilon} \widetilde{v}(t)\right\| \leqslant C$. Следовательно, интерполяционное неравенство

$$
a_{\alpha, \varepsilon_{n}}\left(u^{n}-\widetilde{v}, u^{n}-\widetilde{v}\right) \leqslant C\left\|u^{n}-\widetilde{v}\right\|\left\|A_{\alpha, \varepsilon_{n}}\left(u^{n}-\widetilde{v}\right)\right\|
$$

и оценка (42) дают, что

$$
\max _{t \in[a, b]}\left\|u^{n_{k}}(t)-\widetilde{v}(t)\right\|_{1, \varepsilon_{n_{k}}}^{2} \rightarrow 0, \quad k \rightarrow \infty
$$

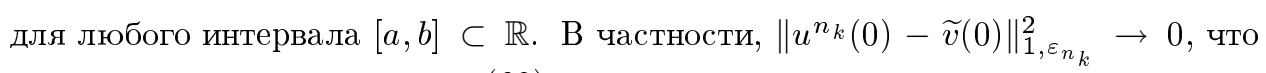
противоречит предположению (69). 
ЗАмЕчАнИЕ 4.1. Из теорем 4.1 и 4.2 для любого $\alpha \in \mathbb{R}$ можно извлечь также следуюшее свойство элементов аттрактора $\mathscr{M}_{\alpha, \varepsilon}$ :

$$
\lim _{\varepsilon \rightarrow 0} \sup _{u \in \mathscr{M}_{\alpha, \varepsilon}} \frac{1}{\varepsilon} \sum_{i=1,2} \int_{\Omega_{i, \varepsilon}}\left|\partial_{z} u_{i}(x, z)\right|^{2} d x d z=0
$$

Подобным образом (за счет (46)) может быть также усилена и теорема 1.2. Кроме того, использование соотношения (43) позволяет получить в теоремах 4.1 и 4.2 полунепрерывность аттрактора в более сильных (чем $H_{\varepsilon}^{1}$ ) нормах.

4.2. Полная непрерывность аттракторов. В случае, когда аттрактор соответствуюшей предельной задачи регулярен (ситуация "общего положения", см. [5] и [13]), можно также установить непрерьвность в $H_{\varepsilon}^{1}$ семейства $\left\{\mathscr{A}_{\alpha, \varepsilon}\right\}_{\varepsilon \in(0,1]}$ при $\varepsilon \rightarrow 0$.

TеOPема 4.3. Пусть $\alpha \geqslant 1$. Пусть все неподвижние точки задачи (10) при $\alpha=1$ ((11) при $\alpha>1)$ гиперболические. Тогда для некоторых $C, \varepsilon_{0}, \sigma>0$ справедливо соотношение

$$
\sup _{v \in \mathscr{A}_{\alpha, \varepsilon}} \inf _{u \in \mathscr{A}_{\alpha}}\|u-v\|_{1, \varepsilon}+\sup _{v \in \mathscr{A}_{\alpha}} \inf _{u \in \mathscr{A}_{\alpha, \varepsilon}}\|u-v\|_{1, \varepsilon} \leqslant C \varepsilon^{\sigma}, \quad 0<\varepsilon \leqslant \varepsilon_{0},
$$

где $\mathscr{A}_{\alpha, \varepsilon}-$ глобальный аттрактор задачи (20)-(23), $\mathscr{A}_{\alpha}-$ глобальный аттрактор соответствующей предельной задачи.

Очевидно, что утверждение теоремы 1.6 вытекает из теоремы 4.3 , замечания 2.2 и соотношений (60) и (67).

ЗАмЕчАниЕ 4.2. В случае $\alpha \geqslant 1$ непосредственной проверкой можно доказать, что

$$
\sum_{i=1,2}\left\|\nabla_{x} \partial_{y} u_{i}\right\|_{L^{2}\left(\Omega_{i}\right)}^{2} \leqslant c_{0} \varepsilon^{2}\left\|A_{\alpha, \varepsilon} u\right\|^{2}, \quad u \in \mathrm{D}\left(A_{\alpha, \varepsilon}\right) .
$$

Поэтому, используя (26) и (42), легко убедиться в том, что

$$
\sup _{u \in \mathscr{A}_{\alpha, \varepsilon}}\|M u-\gamma u\|_{H^{1}(\Gamma) \oplus H^{1}(\Gamma)} \leqslant c \varepsilon, \quad 0<\varepsilon \leqslant 1
$$

где $\gamma$ - оператор следа, $\gamma\left(u_{1} ; u_{2}\right)=\left(u_{1}(x,+0) ; u_{2}(x,-0)\right)$. Это обстоятельство позволяет утверждать, что теоремы 1.4 и 1.6 остаются в силе, если вместо оператора $M^{\varepsilon}$ подставить в (16) и (19) оператор следа $\gamma$.

ДоКАЗАТЕЛЬСТво ТЕОРЕМЫ 4.3 следует хорошо известной схеме (ср. [5], [7], $[8])$. Однако ввиду того, что предельная полугруппа $S_{\alpha}(t)$ действует лишш на некотором подпространстве фазового пространства $H_{\varepsilon}^{1}$, мы не можем непосредственно применять известные общие теоремы о непрерывной зависимости от параметра семейства аттракторов (см., например, [5]) и должны заново получить некоторые оценки в нашей конкретной ситуации. 
Доказательство теоремы проведем лишь для случая $\alpha=1$. Рассуждения в случае $\alpha>1$ аналогичны. В дальнейшем для краткости полагаем $S_{\varepsilon}(t)=S_{1, \varepsilon}(t)$, $S(t)=S_{1}(t)$ и $\mathscr{A}_{\varepsilon}=\mathscr{A}_{1, \varepsilon}, \mathscr{A}=\mathscr{A}_{1}$.

Основным фактом, используемым при доказательстве соотношений вида (70), кроме близости полугрупп $S_{\varepsilon}(t)$ и $S(t)$ является равномерное (по $\varepsilon$ ) экспоненциальное притяжение ограниченных множеств аттракторами $\mathscr{A}_{\varepsilon}$ (см., например, [5; гл. VIII, теорема 2.1]). Доказательство этого факта (см. лемму 4.4) основано на изучении неподвижных точек полугруппы $S_{\varepsilon}(t)$ и свойств ее линеаризаций в окрестности этих точек.

4.2.1. Неподвижные точки $S_{\varepsilon}(t)$. Прежде всего отметим, что имеет место полунепрерывность сверху по норме $H_{\varepsilon}^{1}$ при $\varepsilon \rightarrow 0$ семейства $\left\{\mathscr{N}_{\varepsilon}: \varepsilon \in(0,1]\right\}$ неподвижных точек задачи (20)-(23), т.е.

$$
\lim _{\varepsilon \rightarrow 0} \sup _{u \in \mathcal{N}_{\varepsilon}} \inf _{v \in \mathcal{N}}\|u-v\|_{1, \varepsilon}=0
$$

где $\mathscr{N}$ - множество неподвижных точек задачи (10). Это свойство вытекает из полунепрерывности сверху семейства $\left\{\mathscr{A}_{\alpha, \varepsilon}\right\}_{\varepsilon \in(0,1]}$ (см. теорему 4.1) и может быть установлено тем же методом, что и в [10].

Отметим, что если все неподвижные точки задачи (10) гиперболичны, то их число конечно, $\operatorname{card} \mathscr{N}<\infty$ (см. [5]).

Лемма 4.1. 1) Пусть $v$ - неподвижная гиперболическая точка полугруппь $S(t)$. Для любого $0<q<1$ существует такое $\varepsilon_{0}>0$, что при всех $0<\varepsilon<\varepsilon_{0}$ в окрестности $\mathscr{W}_{\varepsilon} \equiv\left\{u \in H_{\varepsilon}^{1}:\|u-v\|_{1, \varepsilon}<\varepsilon^{q}\right\}$ содержится единственная неподвижная точка $u_{\varepsilon}$ полугруппь $S_{\varepsilon}(t)$.

2) Предположим, что все неподвижные точки задачи (10) гиперболичны. Тогда множество $\mathscr{N}_{\varepsilon}$ конечно, $\operatorname{card} \mathscr{N}_{\varepsilon}=\operatorname{card} \mathscr{N}$ и справедлива оценка

$$
\sup _{v \in \mathscr{N}_{\varepsilon}} \inf _{u \in \mathcal{N}}\|u-v\|_{1, \varepsilon}+\sup _{v \in \mathcal{N}} \inf _{u \in \mathscr{N}_{\varepsilon}}\|u-v\|_{1, \varepsilon} \leqslant \varepsilon^{q}, \quad 0<\varepsilon \leqslant \varepsilon_{0}, \quad 0<q<1 .
$$

ДокаЗАТЕЛЬСтво. Для всех $\delta>0,0<\varepsilon \leqslant 1, u \in H_{\varepsilon}^{1}$ положим

$$
\begin{gathered}
G_{\varepsilon}(u)=u-A_{\varepsilon}^{-1} B(u), \quad \Phi_{\varepsilon}(u)=u-\left(G_{\varepsilon}^{\prime}(v)\right)^{-1} G_{\varepsilon}(u), \\
\Psi_{\varepsilon, \delta}(u)=\eta\left(\frac{\|u-v\|_{1, \varepsilon}}{\delta}\right) \Phi_{\varepsilon}(u)+\left(1-\eta\left(\frac{\|u-v\|_{1, \varepsilon}}{\delta}\right)\right) \Phi_{\varepsilon}(v),
\end{gathered}
$$

где $\eta(r) \in C_{0}^{\infty}(\mathbb{R} ; \mathbb{R}), \operatorname{supp} \eta \subset[-2,2], \eta(r)=1$ при $r \in[-1,1]$. Как и ранее, полагаем $A_{\varepsilon}=A_{1, \varepsilon}$ и $A=A_{1}$.

Покажем, что для любого достаточно малого $\delta>0$ и для любого $0<q<1$ сушествует такое $\varepsilon_{0}>0$, что при всех $0<\varepsilon<\varepsilon_{0}$ отображение $\Psi_{\varepsilon, \delta}$ корректно определено и является сжимающим на пространстве $H_{\varepsilon}^{1}$, причем соответствуюшая неподвижная точка не зависит от $\delta$ и является единственным элементом множества $\mathscr{N}_{\varepsilon} \cap \mathscr{W}_{\varepsilon}$. 
Шаг 1. Покажем, что для всех достаточно малых $\varepsilon>0$

$$
\left\|\left(G_{\varepsilon}^{\prime}(v)\right)^{-1}\right\|_{\mathscr{L}\left(H_{\varepsilon}^{1} ; H_{\varepsilon}^{1}\right)} \leqslant C .
$$

Используя леммы 2.1 и 2.2 , имеем

$$
\left\|\left(A_{\varepsilon}^{-1} B^{\prime}(v)-A^{-1} B^{\prime}(v)\right) M u\right\|_{1, \varepsilon} \leqslant C \varepsilon\left\|B^{\prime}(v) M u\right\| \leqslant C \varepsilon\|M u\|_{1, \varepsilon} .
$$

Поскольку $v \in H^{2}\left(\Gamma_{1}\right) \oplus H^{2}\left(\Gamma_{2}\right)$, то в силу (5) оператор $B^{\prime}(v) \in \mathscr{L}(H ; H)$. Следовательно, с помощью (25) получаем, что

$$
\left\|A_{\varepsilon}^{-1} B^{\prime}(v) N u\right\|_{1, \varepsilon} \leqslant C_{1}\left\|A_{\varepsilon}^{-1 / 2} B^{\prime}(v) N u\right\| \leqslant C_{2} \varepsilon\|N u\|_{1, \varepsilon}
$$

для любого $u \in H_{\varepsilon}^{1}$ и некоторых $C_{i}>0$. Легко видеть (ср., например, [13; лемма 2.5.4]), что оператор $G^{\prime}(v)=I-A^{-1} B^{\prime}(v) M$ фредгольмов. А так как оператор $A-B^{\prime}(v)$ обратим на $M H$ ( $v$ - неподвижная гиперболическая точка), то оператор $G^{\prime}(v)$ обратим на $H_{\varepsilon}^{1}$. Поэтому $(74)$ и $(75)$ дают, что $G_{\varepsilon}^{\prime}(v)$ обратим при всех достаточно малых $\varepsilon$, причем соотношение (73) вьполняется с константой $C$, не зависящей от $\varepsilon$. В частности, отображение $\Psi_{\varepsilon, \delta}$ корректно определено.

Шаг 2. Пусть $0<q<1, \delta>0$. Проверим, что для достаточно малых $\varepsilon>0$ $\Psi_{\varepsilon, \delta}$ отображает шар $B^{\varepsilon}\left(\varepsilon^{q}, v\right) \equiv\left\{u \in H_{\varepsilon}^{1}:\|u-v\|_{1, \varepsilon} \leqslant \varepsilon^{q}\right\}$ в себя.

Так как $v=A^{-1} B(v)$, имеем

$$
v-\Psi_{\varepsilon, \delta}(v)=\left(G_{\varepsilon}^{\prime}(v)\right)^{-1} G_{\varepsilon}(v)=\left(G_{\varepsilon}^{\prime}(v)\right)^{-1}\left(A^{-1}-A_{\varepsilon}^{-1}\right) B(v) .
$$

Поэтому из (73) и леммы 2.1 следует, что

$$
\left\|v-\Psi_{\varepsilon, \delta}(v)\right\|_{1, \varepsilon} \leqslant C \varepsilon
$$

Легко убедиться, что

$$
\Phi_{\varepsilon}(u)-\Phi_{\varepsilon}(w)=\left(G_{\varepsilon}^{\prime}(v)\right)^{-1} A_{\varepsilon}^{-1}\left(B(u)-B(w)-B^{\prime}(v)(u-w)\right)
$$

для всех $u, w \in H_{\varepsilon}^{1}$. Так как $\left\|A_{\varepsilon}^{-1 / 2} y\right\| \leqslant C\|y\|_{H^{-1}}$, то из (73) вытекает, что

$$
\left\|\Phi_{\varepsilon}(u)-\Phi_{\varepsilon}(v)\right\|_{1, \varepsilon} \leqslant C\left\|B(u)-B(v)-B^{\prime}(v)(u-v)\right\|_{H^{-1}} .
$$

Поэтому в силу леммы 2.2

$$
\left\|\Phi_{\varepsilon}(u)-\Phi_{\varepsilon}(v)\right\|_{1, \varepsilon} \leqslant C\left(1+\|u\|_{H^{1}}^{p}+\|v\|_{H^{1}}^{p}\right)\|u-v\|_{H^{1}}^{2} .
$$

Следовательно,

$$
\left\|\Psi_{\varepsilon, \delta}(u)-\Psi_{\varepsilon, \delta}(v)\right\|_{1, \varepsilon} \leqslant \eta\left(\frac{\|u-v\|_{1, \varepsilon}}{\delta}\right)\left\|\Phi_{\varepsilon}(u)-\Phi_{\varepsilon}(v)\right\|_{1, \varepsilon} \leqslant C \varepsilon^{2 q}
$$


для $u \in B^{\varepsilon}\left(\varepsilon^{q}, v\right)$. Поэтому из (76) получаем, что

$$
\left\|v-\Psi_{\varepsilon, \delta}(u)\right\|_{1, \varepsilon} \leqslant C\left(\varepsilon+\varepsilon^{2 q}\right) \leqslant \varepsilon^{q}, \quad u \in B^{\varepsilon}\left(\varepsilon^{q}, v\right),
$$

для всех $\varepsilon \in\left(0, \varepsilon_{0}\right]$.

Шаг 3. Отображение $\Psi_{\varepsilon, \delta}$ является сжимаюшим на $H_{\varepsilon}^{1}$ при всех достаточно малых $\delta>0$ и $\varepsilon \in\left(0, \varepsilon_{0}\right]$. Действительно, очевидно, что

$\Psi_{\varepsilon, \delta}^{\prime}(u) h=\eta\left(\frac{\|u-v\|_{1, \varepsilon}}{\delta}\right) \Phi_{\varepsilon}^{\prime}(u) h+\frac{1}{\delta} \eta^{\prime}\left(\frac{\|u-v\|_{1, \varepsilon}}{\delta}\right) \frac{(u-v, h)_{1, \varepsilon}}{\|u-v\|_{1, \varepsilon}}\left(\Phi_{\varepsilon}(u)-\Phi_{\varepsilon}(v)\right)$

для любого $u \in H_{\varepsilon}^{1}$. Из (78) вытекает, что

$$
\left\|\Phi_{\varepsilon}(u)-\Phi_{\varepsilon}(v)\right\|_{1, \varepsilon} \leqslant C \delta^{2}
$$

при условии, что $\|u-v\|_{1, \varepsilon} \leqslant 2 \delta, \delta<1$. Из (77) следует, что

$$
\Phi_{\varepsilon}^{\prime}(u)=\left(G_{\varepsilon}^{\prime}(v)\right)^{-1} A_{\varepsilon}^{-1}\left(B^{\prime}(u)-B^{\prime}(v)\right) .
$$

Поэтому легко видеть, что $\left\|\Phi_{\varepsilon}^{\prime}(u)\right\|_{\mathscr{L}\left(H_{\varepsilon}^{1} ; H_{\varepsilon}^{1}\right)} \leqslant C \delta$, если $\|u-v\|_{1, \varepsilon} \leqslant 2 \delta$. Следовательно, $\left\|\Psi_{\varepsilon, \delta}^{\prime}(u)\right\|_{\mathscr{L}\left(H_{\varepsilon}^{1} ; H_{\varepsilon}^{1}\right)} \leqslant C \delta$ для всех $u \in H_{\varepsilon}^{1}$. Отсюда вытекает сжимаемость отображения $\Psi_{\varepsilon, \delta}$ в $H_{\varepsilon}^{1}$ для достаточно малых $\delta>0$. Так как $\Psi_{\varepsilon, \delta}$ отображает $B^{\varepsilon}\left(\varepsilon^{q}, v\right)$ в себя, то единственная неподвижная точка $u_{\varepsilon, \delta}$ для $\Psi_{\varepsilon, \delta}$ лежит в $B^{\varepsilon}\left(\varepsilon^{q}, v\right)$. В случае, когда $\varepsilon^{q}<\delta$, имеем $u_{\varepsilon, \delta} \in \mathscr{N}_{\varepsilon}$.

Докажем вторую часть леммы. Из (71) вытекает, что для достаточно малых $\varepsilon$ каждая неподвижная точка $u_{\varepsilon} \in \mathscr{N}_{\varepsilon}$ попадает в $\delta$-окрестность некоторой точки $v \in \mathscr{N}$, т.е. $u_{\varepsilon}$ является единственной неподвижной точкой отображения $\Psi_{\varepsilon, \delta}$ для некоторого $\delta$. При этом можно считать, что $\delta$-окрестности различных неподвижных точек из $\mathscr{N}$ не пересекаются. Следовательно, $u_{\varepsilon} \in \mathscr{W}_{\varepsilon}$ и справедлива оценка (72). Лемма доказана.

\subsection{2. Равномерное экспоненциальное притяжение.}

Лемма 4.2. Для любых $v \in M H_{\varepsilon}^{1}, n \in \mathbb{N}, \delta>0$ существуют такие $\varepsilon_{0}>0$, $\rho>0$, что для всех $u \in H_{\varepsilon}^{1},\|u-v\|_{1, \varepsilon} \leqslant \rho, 0<\varepsilon \leqslant \varepsilon_{0}$, выполняется соотношение

$$
\max _{1 \leqslant j \leqslant n}\left|\lambda_{j, \varepsilon}(u)-\lambda_{j}(v)\right| \leqslant \delta,
$$

где $\lambda_{1, \varepsilon}(u) \leqslant \lambda_{2, \varepsilon}(u) \leqslant \cdots\left(\lambda_{1}(v) \leqslant \lambda_{2}(v) \leqslant \cdots\right)$ - собственнше числа операmopa $A_{\varepsilon}-B^{\prime}(u)\left(A-B^{\prime}(v)\right)$. 
ДокаЗАТЕЛЬСтво. Рассмотрим операторы

$$
\begin{array}{cc}
C_{\varepsilon}(u) g=\left(A_{\varepsilon}-B^{\prime}(u)+b\right) g, & g \in \mathrm{D}\left(A_{\varepsilon}\right), \\
C(v) h=\left(A-B^{\prime}(v)+b\right) h, & h \in \mathrm{D}(A),
\end{array}
$$

где $b>0$ достаточно велико. Легко проверить (см. [16; гл. 5, теорема 4.11]), что $C_{\varepsilon}(u)(C(v))$ - самосопряжен и положителен в пространстве $H(M H)$. Пусть $c_{\varepsilon}^{u}(g, h)$ и $c^{v}(g, h)$ - билинейные формы на $H_{\varepsilon}^{1}$, соответствующие операторам $C_{\varepsilon}(u)$ и $C(v)$. Рассуждая, как и при доказательстве леммы 2.1 , находим, что

$$
\begin{aligned}
& c_{\varepsilon}^{u}\left(\left(C_{\varepsilon}(u)^{-1}-C(v)^{-1}\right) g, h\right)=\left(\left(B^{\prime}(u)-B^{\prime}(v)\right) C(v)^{-1} g, h\right)_{H} \\
& \quad+\sum_{i=1}^{2}(-1)^{i} \int_{\Gamma} k(x)\left(h_{i}(x, 0)-M_{i} h_{i}\right)\left(\left(C(v)^{-1} g\right)_{1}-\left(C(v)^{-1} g\right)_{2}\right) d x
\end{aligned}
$$

для всех $g \in M H \cap H_{\varepsilon}^{1}$ и $h \in H_{\varepsilon}^{1}$. Поэтому, используя лемму 2.2, как и ранее, получаем, что

$$
\begin{aligned}
& \left|c_{\varepsilon}^{u}\left(\left(C_{\varepsilon}(u)^{-1}-C(v)^{-1}\right) g, h\right)\right| \leqslant C \varepsilon\|h\|_{1, \varepsilon}\left\|C(v)^{-1} g\right\| \\
& +C\|h\|_{1, \varepsilon}\left(1+\|u\|_{1, \varepsilon}^{p}+\|v\|_{1, \varepsilon}^{p}\right)\|u-v\|_{1, \varepsilon}\|g\| .
\end{aligned}
$$

Так как $c_{\varepsilon}^{u}(h, h) \geqslant \beta\|h\|_{1, \varepsilon}^{2}, \beta>0$, после подстановки $h=\left(C_{\varepsilon}(u)^{-1}-C(v)^{-1}\right) g$, получаем, что

$$
\left\|\left(C_{\varepsilon}(u)^{-1}-C(v)^{-1}\right) g\right\|_{1, \varepsilon} \leqslant C\left(\varepsilon+\left(1+\|u\|_{1, \varepsilon}^{p}+\|v\|_{1, \varepsilon}^{p}\right)\|u-v\|_{1, \varepsilon}\right)\|g\| .
$$

Полученное соотношение позволяет применить к $C_{\varepsilon}(u)$ теорему о спектре и собственных векторах сингулярно возмушенного семейства операторов (см. [17; теорема 1]) и получить утверждение леммы.

ЛЕмма 4.3. Пусть $U_{\varepsilon, R}=\left\{u \in H_{\varepsilon}^{1}:\|u\|_{1, \varepsilon} \leqslant R\right\}$ - поглощающее множество для полугруппь $S_{\varepsilon}(t), \varepsilon \in(0,1]$. Для любого $\delta>0$ существует $T=T(\delta)>0$ такое, что при всех $0<\varepsilon \leqslant 1, u \in U_{\varepsilon, R}$ найдется $t \in[0, T]$, для которого выполняется соотношение

$$
\inf _{w \in \mathcal{N}_{\varepsilon}}\left\|S_{\varepsilon}(t) u-w\right\|_{1, \varepsilon}<\delta
$$

ДокАЗАТЕЛЬСТво. Покажем сначала, что для любого $\delta>0$ сушествуют $\varepsilon_{0} \in$ $(0,1]$ и $T_{0}>0$ такие, что для всех $0<\varepsilon \leqslant \varepsilon_{0}, u \in U_{\varepsilon, R}$ выполняется соотношение

$$
\inf _{v \in \mathcal{N}}\left\|S_{\varepsilon}(t) u-v\right\|_{1, \varepsilon}<\delta
$$

для некоторого $t \in\left[0, T_{0}\right]$. Если это не так, то сушествуют число $\delta>0$ и последовательности $\left\{\varepsilon_{n}\right\}_{n=1}^{\infty}, \lim _{n \rightarrow \infty} \varepsilon_{n}=0,\left\{u^{n}\right\}_{n=1}^{\infty} \subset U_{\varepsilon, R}$ такие, что

$$
\inf _{v \in \mathcal{N}}\left\|S_{\varepsilon_{n}}(t) u^{n}-v\right\|_{1, \varepsilon_{n}} \geqslant \delta, \quad t \in[0, n], \quad n \in \mathbb{N} .
$$


В силу (47) на любом интервале $t \in[1, m], m \in \mathbb{N}$, имеем

$$
\begin{aligned}
\inf _{v \in \mathcal{N}}\left\|S(t) M u^{n}-v\right\|_{1, \varepsilon_{n}} \geqslant & \inf _{v \in \mathcal{N}}\left\|S_{\varepsilon_{n}}(t) u^{n}-v\right\|_{1, \varepsilon_{n}} \\
& -\left\|S(t) M u^{n}-S_{\varepsilon_{n}}(t) u^{n}\right\|_{1, \varepsilon_{n}} \geqslant \frac{\delta}{2}
\end{aligned}
$$

для всех достаточно больших $n \in \mathbb{N}$. Последовательность $S(1) M u^{n}$, очевидно, ограничена в $H^{2}(\Gamma) \oplus H^{2}(\Gamma)$, а значит, содержит сходящуюся в $H^{1}(\Gamma) \oplus H^{1}(\Gamma)$ подпоследовательность. Тогда для соответствующей предельной точки $w$ имеем

$$
\inf _{v \in \mathcal{N}}\|S(t-1) w-v\|_{H^{1}(\Gamma) \oplus H^{1}(\Gamma)} \geqslant \frac{\delta}{2}, \quad t \geqslant 1
$$

что противоречит тому, что (10) имеет функционал Ляпунова на фазовом пространстве и любая полутраектория должна стремиться к множеству неподвижных точек. Следовательно, неравенство (80) справедливо с какими-то $\varepsilon_{0} \in(0,1]$ и $T_{0}>0$.

В силу $(72)$ и $(80)$ для любого $\delta>0$ сушествует $\varepsilon_{0} \in(0,1]$ такое, что при всех $0<\varepsilon<\varepsilon_{0}, u \in U_{\varepsilon, R}$ и некотором $t \in\left[0, T_{0}\right]$ выполняется соотношение

$$
\inf _{w \in \mathcal{N}_{\varepsilon}}\left\|S_{\varepsilon}(t) u-w\right\|_{1, \varepsilon} \leqslant \inf _{v \in \mathcal{N}}\left\|S_{\varepsilon}(t) u-v\right\|_{1, \varepsilon}+\sup _{v \in \mathcal{N}} \inf _{w \in \mathscr{N}_{\varepsilon}}\|v-w\|_{1, \varepsilon}<\delta
$$

Семейство полугрупा $S_{\varepsilon}(t)$ при $\varepsilon_{0} \leqslant \varepsilon \leqslant 1$ обладает функционалом Ляпунова $\varphi_{\varepsilon}(u)$, непрерывным на $H_{\varepsilon}^{1} \times\left[\varepsilon_{0}, 1\right]$ (см. $\left.(36)\right)$, и удовлетворяет оценке (41). Применяя теорему 2.1 , легко проверить, что множество $U=\bigcup_{\varepsilon \in\left[\varepsilon_{0}, 1\right]} S_{\varepsilon}(1) U_{\varepsilon, R}$ компактно в $H_{\varepsilon}^{1}$ и является поглощающим (равномерно по $\varepsilon$ ) для всех полугрупп $S_{\varepsilon}(t)$ с $\varepsilon_{0} \leqslant \varepsilon \leqslant 1$. Отсюда легко вывести (см., например, [5; гл. IX, $\S 1$, лемма 1]), что для любого $\delta>0$ найдется такое $T_{1}>0$, что при $u \in U$ для некоторого $t \in\left[0, T_{1}\right]$ имеет место (79). Учитывая оценку (81) и полагая $T=T_{0}+T_{1}+1$, получаем утверждение леммы.

Лемма 4.4. Существуют $\varepsilon_{0}>0$ и константы $C>0 u \eta>0$ такие, что

$$
\sup _{u \in U_{\varepsilon, R}} \inf _{w \in \mathscr{A}_{\varepsilon}}\left\|S_{\varepsilon}(t) u-w\right\|_{1, \varepsilon} \leqslant C e^{-\eta t}, \quad t \geqslant 0, \quad 0<\varepsilon \leqslant \varepsilon_{0}
$$

где $U_{\varepsilon, R}=\left\{u \in H_{\varepsilon}^{1}:\|u\|_{1, \varepsilon} \leqslant R\right\}-$ поглощающее множсество для полугруппь $S_{\varepsilon}(t)(c \mathcal{M} .(33))$.

ДокАЗАТЕЛЬСТво. В силу леммы 4.1 множество $\mathscr{N}_{\varepsilon}$ неподвижных точек для $S_{\varepsilon}(t)$ при $0<\varepsilon \leqslant \varepsilon_{0}$ является конечным. Из леммы 4.2 вытекает, что эти точки гиперболичны. Все полугруппы $S_{\varepsilon}(t), 0<\varepsilon \leqslant \varepsilon_{0}$, диссипативны, компактны, обладают функционалом Ляпунова на пространстве $H_{\varepsilon}^{1}$ и удовлетворяют оценке (41). Кроме того, применяя теорему $1[5 ;$ гл. $7, \S 1]$ и свойства дифференцируемости отображения $B$ (лемма 2.2 ), нетрудно убедиться, что $S_{\varepsilon}(t)$ принадлежит 
классу $C^{1}\left(H_{\varepsilon}^{1}\right)$ и соответствуюшая производная $\Phi$ реше $\left[S_{\varepsilon}^{\prime}(t) u\right]$ является эволюционным оператором задачи

$$
\partial_{t} w+A_{\varepsilon} w=B^{\prime}\left(S_{\varepsilon}(t) u\right) w
$$

При этом $\left[S_{\varepsilon}^{\prime}(t) u\right]$ удовлетворяет оценкам, аналогичным неравенствам (41) и (47) (и получаемым аналогичным образом), а именно,

$$
\begin{gathered}
\left\|\left[S_{\varepsilon}^{\prime}(t) u_{1}\right]-\left[S_{\varepsilon}^{\prime}(t) u_{2}\right]\right\|_{\mathscr{L}\left(H_{\varepsilon}^{1} ; H_{\varepsilon}^{1}\right)} \leqslant C_{1} e^{L_{1} t}\left\|u_{1}-u_{2}\right\|_{1, \varepsilon} \\
t^{1 / 2}\left\|\left[S_{\varepsilon}^{\prime}(t) u_{1}\right] u_{2}-\left[S^{\prime}(t) M u_{1}\right] M u_{2}\right\|_{1, \varepsilon}^{2} \leqslant C_{2} \varepsilon e^{L_{2} t}
\end{gathered}
$$

где $t \geqslant 0, \varepsilon \in(0,1],\left\|u_{1}\right\|_{1, \varepsilon},\left\|u_{2}\right\|_{\varepsilon} \leqslant r$, а константы $C_{1}, C_{2}, L_{1}, L_{2}$ зависят только от $r$. Поэтому можно применить теорему 1 [5; гл. $\mathrm{V}, \S 7]$ и получить, что для всех достаточно малых $\varepsilon>0$ сушествуют такие $C_{\varepsilon}, \eta_{\varepsilon}$, что (82) выполняется с $C=C_{\varepsilon}$ и $\eta=\eta_{\varepsilon}$. Однако постоянная $\eta_{\varepsilon}$ зависит только от числа $K$ в оценке (41) и от некоторого конечного (и не зависящего от $\varepsilon$ ) числа собственных значений операторов $A_{\varepsilon}-B^{\prime}(u)$ с $u \in \mathscr{N}_{\varepsilon}$ (которые близки по лемме 4.2 к соответствуюшим собственным значениям операторов $A-B^{\prime}(v)$ с $\left.v \in \mathscr{N}\right)($ см. [5; гл. V, $\left.\S 7]\right)$. Постоянная $C_{\varepsilon}$ зависит от времени $T=T(\delta)$ из леммы 4.3 и от равномерно по $\varepsilon \in\left(0, \varepsilon_{0}\right]$ ограниченных характеристик полугруппы $S_{\varepsilon}(t)$, а именно, от постоянных в соотношениях (83) и в оценках, приведенных в теоремах 2.1 и 3.1 (см. [5], там же). Следовательно, соотношение (82) выполняется с $C=\sup _{0<\varepsilon \leqslant \varepsilon_{0}} C_{\varepsilon}<\infty$ и $\eta=\inf _{0<\varepsilon \leqslant \varepsilon_{0}} \eta_{\varepsilon}>0$.

Для завершения доказательства теоремы 4.3 осталось воспользоваться рассуждениями, приведенньми при доказательстве теоремы 1 [5; гл. VIII, §2]. Отметим, что формально эта теорема неприменима в нашей ситуации (например, из-за того, что фазовое пространство $H_{\varepsilon}^{1}$ зависит от $\varepsilon$ и предельная полугруппа $S(t)$ определена лиш на некотором подпространстве из $\left.H_{\varepsilon}^{1}\right)$. Однако соответствуюшая модификация рассуждений не составляет особого труда.

\section{Список литературы}

1. Hale J., Raugel G. Reaction-diffusion equation on thin domains // J. Math. Pures Appl. (9). 1992. V. 71. P. 33-95.

2. Hale J., Raugel G. A reaction-diffusion equation on a thin L-shaped domain // Proc. Roy. Soc. Edinburgh Sect. A. 1995. V. 125. P. 283-327.

3. Ciuperca I. S. Reaction-diffusion equations on thin domains with varying order of thinness // J. Differential Equations. 1996. V. 126. P. 244-291.

4. Prizzi M., Rybakowski K.P. Some recent results on thin domain problem // Topol. Methods Nonlinear Anal. 1999. V. 14. P. 239-255.

5. Бабин А. В., Вишик М. И. Аттракторы эволюционных уравнений. М.: Наука, 1989.

6. Hale J. Asymptotic behavior of dissipative systems. Providence, RI: Amer. Math. Soc., 1988.

7. Капитанский Л. В, Костин И. Н. Аттракторы нелинейных эволюционных уравнений и их аппроксимаций // Алгебра и анализ. 1990. Т. 2. № 1. С. 114-140.

8. Костин И. Н. Регулярный подход в задаче об аттракторах сингулярно возмущенных уравнений // Записки научн. сем. ЛОМИ. 1990. Т. 181. С. 93-131. 
9. Boutet de Monvel L., Chueshov I. D., Khruslov E. Ya. Homogenization of attractors for semilinear parabolic equations on manifolds with complicated microstructure // Ann. Mat. Pura Appl. (4). 1997. V. 162. P. 297-322.

10. Панкратов Л. С., Чуешов И. Д. Усреднение аттракторов нелинейных гиперболических уравнений с асимптотически вырождающимися коэффициентами // Матем. сб. 1999. T. 190. № 9. C. 99-126.

11. Bourgeat A., Chueshov I. D., Pankratov L.S. Homogenization of attractors of semilinear parabolic equations in domains with spherical traps // C. R. Acad. Sci. Paris Sér. I Math. 1999. V. 329. P. 581-587.

12. Вишик М.И., Чепьжсов В.В. Усреднение траекторных аттракторов эволюционных уравнений с быстро осциллирующими членами // Матем. сб. 2001. Т. 192. № 1. С. 13-50.

13. Чуешов И. Д. Введение в теорию бесконечномерных диссипативных систем. Харьков: Акта, 1999; http://www.emis.de/monographs/Chueshov/.

14. Rekalo A. Asymptotic behaviour of solutions of nonlinear parabolic equations on two-layer thin domains // Nonlinear Anal. 2003. V. 52. P. 1393-1410.

15. Хенри Д. Геометрическая теория полулинейных параболических уравнений. М.: Мир, 1985.

16. Като Т. Теория возмущений линейных операторов. М.: Мир, 1972.

17. Иосифьян Г. А., Олейник О. А., Шамаев А. С. О предельномповедении спектра последовательности операторов, заданных в различных гильбертовых пространствах // УМН. 1989. T. 44. № 3. C. 157-158.

Харьковский университет, Украина

Поступила в редакцию

15.01 .2003 